\title{
A synthetic antibiotic scaffold effective against multidrug-resistant bacterial pathogens.
}

Matthew J. Mitcheltree ${ }^{1, \dagger}$, Amarnath Pisipati ${ }^{1, \dagger}$, Egor A. Syroegin ${ }^{2, \dagger}$, Katherine J. Silvestre ${ }^{1}$, Dorota Klepacki $^{3}$, Jeremy D. Mason ${ }^{1}$, Daniel W. Terwilliger ${ }^{1}$, Giambattista Testolin ${ }^{1}$, Aditya R. Pote $^{1}$, Kelvin J.Y. Wu ${ }^{1}$, Richard Porter Ladley ${ }^{1}$, Kelly Chatman ${ }^{4}$, Alexander S. Mankin ${ }^{3}$, Yury S. Polikanov $^{2, *}$, and Andrew G. Myers ${ }^{1, *}$

${ }^{1}$ Department of Chemistry and Chemical Biology, Harvard University, Cambridge, MA 02138, USA

${ }^{2}$ Department of Biological Sciences, University of Illinois at Chicago, Chicago, IL 60607, USA

${ }^{3}$ Department of Pharmaceutical Sciences, University of Illinois at Chicago, Chicago, IL 60607, USA

${ }^{4}$ Harvard Center for Mass Spectrometry, Harvard University, Cambridge, MA 02138, USA

${ }^{\dagger}$ Authors contributed equally to this work

* To whom correspondence should be addressed:

E-mail: yuryp@uic.edu (Y.S.P.)

E-mail: myers@chemistry.harvard.edu (A.G.M.) 


\section{SUMMARY}

The dearth of new medicines effective against antibiotic-resistant bacteria presents a growing global public health concern. For more than five decades, the search for new antibiotics has relied heavily upon the chemical modification of natural products (semi-synthesis), a method illequipped to combat rapidly evolving resistance threats. Semi-synthetic modifications are typically of limited scope within polyfunctional antibiotics, usually increase molecular weight, and seldom permit modifications of the underlying scaffold. When properly designed, fully synthetic routes can easily address these shortcomings. Here we report the structure-guided design and componentbased synthesis of a rigid oxepanoproline scaffold which, when linked to the aminooctose residue of clindamycin, produces an antibiotic of exceptional potency and spectrum of activity, here named iboxamycin. Iboxamycin is effective in strains expressing Erm and Cfr rRNA methyltransferase enzymes, products of genes that confer resistance to all clinically relevant antibiotics targeting the large ribosomal subunit, namely macrolides, lincosamides, phenicols, oxazolidinones, pleuromutilins, and streptogramins. X-ray crystallographic studies of iboxamycin in complex with the native $70 \mathrm{~S}$ bacterial ribosome, as well as the Erm-methylated $70 \mathrm{~S}$ ribosome, uncover the structural basis for this enhanced activity, including an unforeseen and unprecedented displacement of $\mathrm{m}_{2}^{6} \mathrm{~A} 2058$ upon antibiotic binding. In mice, iboxamycin is orally bioavailable, safe, and effective in treating bacterial infections, testifying to the capacity for chemical synthesis to provide new antibiotics in an era of rising resistance. 


\section{BODY TEXT}

\section{Introduction}

The rapid rise and widespread dissemination of bacteria resistant to most or all known antibiotics raises concerns of an impending global infectious-disease crisis. Any plan to address the problem will be predicated upon the discovery of new antibiotics effective against modern bacterial pathogens, but opinions diverge on which strategies can best achieve these discoveries. ${ }^{1}$ For decades, natural products have served as both conceptual and material starting points for antibiotics discovery, but making specific chemical modifications to structurally complex natural products is inherently challenging, and the pace of drug discovery by semi-synthesis, as it is known, has slowed markedly ${ }^{2}$. In an alternative approach, the development of fully synthetic platforms to construct tetracycline ${ }^{3}$, macrolide ${ }^{4}$, and group A streptogramin ${ }^{5}$ antibiotics has permitted deepseated structural modifications not achievable through semi-synthesis ${ }^{6}$. These technologies allow chemists to envision and reduce to practice an almost limitless array of design hypotheses. In this work, we employ component-based chemical synthesis to achieve a substantive re-scaffolding of the aminoacyl hemisphere of lincosamide antibiotics, giving rise to an antibacterial agent radically divergent from its parent class with respect to potency, spectrum of activity, and capacity to overcome resistance mechanisms that render clinically approved lincosamides (and many other antibiotics) ineffective.

The ribosome is a major antibiotic target within bacteria, and lincosamides are one of several ribosome-targeting antibiotic classes that have proven essential to the modern pharmacopeia ${ }^{7,8}$. Researchers at the Upjohn Company isolated the founding member of the class, lincomycin (1, Fig. 1a), in 1963 from the fermentation broth of a streptomycete found in a Nebraskan soil sample . The antibiotic was found useful for the treatment of streptococcal, pneumococcal, and staphylococcal infections. In an early semi-synthetic modification of $\mathbf{1}$, stereoinvertive deoxychlorination at position $\mathrm{C} 7$ of the aminosugar residue afforded the antibiotic now known as clindamycin (2, Fig. 1a), a molecule with improved pharmacokinetic properties and an enhanced spectrum of activity ${ }^{10}$. Clindamycin has largely replaced lincomycin in human medicine ${ }^{11}$ though in latter years recognition of the propensity of clindamycin to promote Clostridioides difficile colitis has constrained its use. In the decades after clindamycin's 1970 FDA approval, semisynthetic and fully synthetic approaches to the discovery of new lincosamide antibiotics were 
explored, giving rise to candidates containing six- and seven-membered aminoacyl residues, each with an expanded spectrum of activity (e.g., 3, Fig. 1a) ${ }^{12,13,14}$. More recently, modifications of the aminosugar residue by semi-synthesis have been reported, expanding coverage to certain multidrug-resistant Gram-positive bacteria ${ }^{15,16,17}$.

Bacterial pathogens resistant to clindamycin are now globally distributed both in the community and nosocomially. Acquired resistance to clindamycin most commonly occurs through $\mathrm{N}^{6}$ dimethylation of 23S rRNA residue A2058 (E. coli nucleotide numbering is used throughout this report) by erythromycin resistance (Erm) rRNA methyltransferases ${ }^{18,19,20}$. Encoded by erm genes, these enzymes give rise to macrolide, lincosamide, and streptogramin B $\left(\mathrm{MLS}_{\mathrm{B}}\right)$ cross-resistance; expression of these genes may be constitutive (c-erm), or inducible upon exposure to macrolide antibiotics (i-erm $)^{21,22}$. The prevalence of erm genes among clinical streptococcal and staphylococcal isolates has led the CDC to list $\mathrm{MLS}_{\mathrm{B}}$ pathogens among its most pressing threats in their 2019 report $^{23}$. Recently, the emergence of another rRNA methyltransferase, Cfr, encoded by the plasmid- or transposon-borne, horizontally transferrable gene $c f r$ was reported. ${ }^{24} \mathrm{Cfr}$ methylates C8 of the 23S rRNA residue A2503 and confers resistance to phenicol, lincosamide, oxazolidinone, pleuromutilin, and streptogramin A $\left(\mathrm{PhLOPS}_{\mathrm{A}}\right)^{25,26}$ antibiotics as well as 16membered macrolides ${ }^{27}$. The prevalence of erm and $c f r$ resistance genotypes in staphylococcal, streptococcal, and enterococcal clinical isolates, together with clindamycin's risk of $C$. difficile colitis challenge the continued utility of this antibiotic in patients.

\section{Discovery of oxepanoprolinamides}

To enable full structural exploration of the lincosamides, we developed modular routes for the synthesis of both the aminooctose and aminoacyl residues, then combined these components by simple amide-bond formation to produce a collection of $>500$ antibacterial candidates ${ }^{28,29,30,31}$. Although a wide array of structural changes were examined, we observed and here report that bicyclic scaffold modifications of the aminoacyl hemisphere in combination with the aminooctose residue of clindamycin were especially advantageous with respect to antibacterial activity, notably among clindamycin-resistant strains. Briefly, the bicyclic scaffold of oxepanoprolinamide 1 (OPP1) was conceived in an initial design hypothesis in which we sought to rigidify the $\mathrm{C} \gamma$-endo proline ring pucker that clindamycin adopts when bound to the bacterial ribosome and at the same time enable substitution of the 7'-position of the oxepane ring, so as to overlay and extend the elongated 
$4^{\prime}-n$-propyl chain of clindamycin in its bound conformation ${ }^{32}$. These explorations were both inspired and made feasible by a highly stereoselective aldol addition reaction developed for a key fragment coupling in the synthesis of macrolide antibiotics ${ }^{4}$. Thus, enolization of $(R, R)-\mathbf{4}$, a chiral glycine equivalent ${ }^{33}$, followed by addition of the bis-electrophile $\mathbf{5}$ led to sequential syn-aldol addition to set the $2^{\prime}, 3^{\prime}$-stereodiad within the target, then spontaneous intramolecular $\mathrm{N}$-alkylation, furnishing the $\beta$-hydroxy proline derivative $\mathbf{6}$ in a single operation and in amounts more than sufficient to supply our early discovery efforts (64\% yield, 2.3-g scale). A three-step sequence involving cleavage of the chiral auxiliary (with $>99 \%$ recovery of pseudoephenamine), N-Boc protection, and esterification provided the suitably protected intermediate 7 in $85 \%$ overall yield. Construction of the oxepene ring was then achieved through palladium-catalyzed O-allylation followed by ring-closing metathesis using the first-generation Hoveyda-Grubbs catalyst ${ }^{34}$. The target OPP-1 was assembled following an established sequence of saponification, amide coupling to the aminosugar component (7-chloro-1-methylthio-lincosamine, 7-Cl-MTL) ${ }^{35}$ and $\mathrm{N}$-Boc removal $^{14}$. 
a
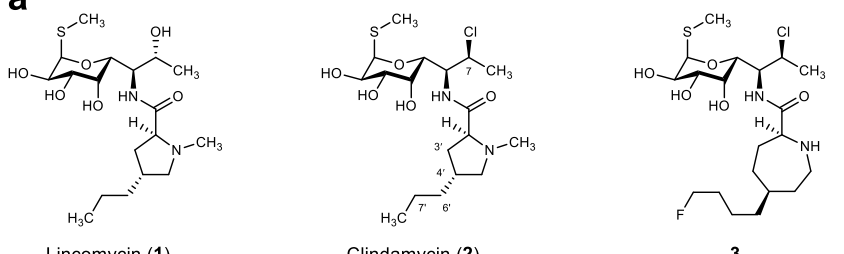

Lincomycin (1) Fermentation product
FDA approval 1964

Clindamycin (2) 1 step from lincomycin

3

Fully synthetic southern half

b

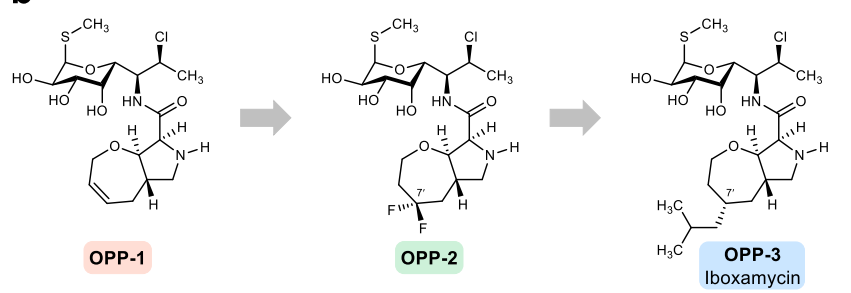

C<smiles>C=CC[C@H](COP(=O)(O)c1c(C)cc(C)cc1C)C(=O)NC</smiles><smiles>[Y]C(=O)C1NCC(CC=C)C1O</smiles>
$\sqrt{b, c, d}$

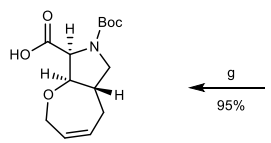

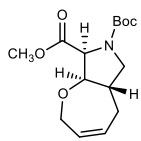<smiles>C=CC[C@H]1CN(C)[C@H](C(=O)OC)[C@H]1O</smiles>

OPP-1

$\downarrow_{74 \%}^{j}$
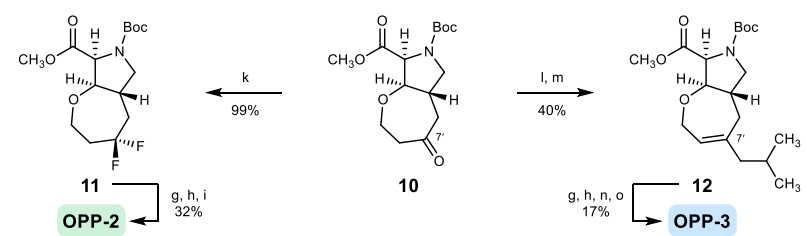

Figure 1 | Evolution of a novel antibiotic scaffold. a, Chemical structures of the clinically approved lincosamide antibiotics lincomycin and clindamycin, as well as a broad-spectrum preclinical antibiotic discovered by Vicuron scientists. b, The design progression of oxepanoprolinamide antibiotics. Conformational constraint of the C4' substituent within clindamycin, together with lipophilic decoration of the resulting oxepanoprolyl 7' position provided substantial improvements in activity and spectrum of action. c, Synthesis of oxepanoprolinamides OPP-1, OPP-2, and iboxamycin (OPP-3). Reagents and conditions are as follows: (a) LiHMDS, LiCl; (b) NaOH, >99\% recovery of $(R, R)$-pseudoephenamine; (c) Boc ${ }_{2} \mathrm{O}$; (d) $\mathrm{MeI}, \mathrm{Cs}_{2} \mathrm{CO}_{3}$; (e) allyl ethyl carbonate, $\mathrm{Pd}\left(\mathrm{PPh}_{3}\right)_{4}$; (f) Hoveyda-Grubbs catalyst I; (g) LiOH; (h) 7-Cl-MTL, HATU, Et ${ }^{i} \operatorname{Pr}_{2} \mathrm{~N}$; (i) TFA; (j) Pd(OAc) $)_{2}$, benzoquinone, HBF 4 (aq.); (k) DAST; (l) LiHMDS, Comins' reagent, $51 \%$ (plus, separately, $31 \% \Delta^{6^{\prime}}$ regioisomer); (m) ${ }^{i} \mathrm{BuMgCl}, \mathrm{Fe}(\operatorname{acac})_{3} ;(\mathrm{n}) \mathrm{H}_{2}, \mathrm{Pd}(\mathrm{OH})_{2} / \mathrm{C}$, 1:1 dr; (o) BSTFA, TMSI, 17\% over 2 steps (plus, separately, the 7'R epimer; 17\% over 2 steps).

Microbiological testing of OPP-1 showed it to be comparable in activity to clindamycin (Extended Data Fig. 2). Substitution of position 7' of the oxepene ring was prioritized next as molecular modeling had suggested that substituents there would project toward the hydrophobic 
cleft formed by 23S rRNA residues A2451 and C2452 (Extended Data Fig. 1a). This cleft ordinarily accommodates the $\alpha$-side-chain residues of aminoacylated A-site tRNAs in staging for amide bond formation with the nascent peptide. Auspiciously, Wacker oxidation of intermediate 8 using Grubbs' protocol proceeded with complete regioselectivity to provide the 7'-keto product 10 in $74 \%$ yield $^{36}$; this selectivity is thought to arise from the $\sigma$-withdrawing effect of the oxygen atom embedded within the 7-membered ring. Deoxydifluorination of intermediate $\mathbf{8}$ with $N, N$ diethylaminosulfur trifluoride (DAST) followed by the same 3-step sequence described earlier provided oxepanoprolinamide OPP-2, which exhibited substantially improved activity against multidrug-resistant Gram-positive strains (Streptococcus pneumoniae cErmA; Streptococcus pyogenes cErmB), as well as against Klebsiella pneumoniae (Extended Data Fig. 2), lending support to our design strategy. Alkyl (and aryl) substitution of position 7' was made possible by non-regioselective enolization of $\mathbf{1 0}$ with lithium hexamethyldisilazide (LiHMDS), followed by triflylation with Comins' reagent. The resulting regioisomeric vinyl triflates were readily separated by flash-column chromatography, and both served as flexible intermediates for metal-catalyzed cross-coupling with aryl and alkyl nucleophiles. For example, iron-catalyzed coupling of the $\Delta^{7^{\prime}}$ vinyl triflate with isobutylmagnesium chloride ${ }^{37}$ provided intermediate 12, which upon saponification, amide coupling, non-diastereoselective olefin saturation ( $\mathrm{dr} \sim 1: 1), N$-Boc removal, and purification by HPLC provided separately OPP-3 (Fig. 1c) and its less active 7'- $\beta$ stereoisomer epi-OPP-3. We found that, generally, $7^{\prime}$ - $\alpha$-alkyl analogs were more active than their unsaturated and 7'- $\beta$ stereoisomeric congeners (Extended Data Fig. 2). This sequence provided a viable discovery route to OPP-3 - henceforth termed "iboxamycin" - and other 7'-substituted analogs in amounts sufficient for minimum inhibitory concentration (MIC) analyses but was not capable of providing the quantities of material necessary for in vivo evaluations of iboxamycin in animal infection models. For this purpose, an entirely different sequence to the specific target iboxamycin was devised, one which has provided iboxamycin in multigram amounts and could potentially provide the basis of a manufacturing route ${ }^{38}$.

\section{Iboxamycin overcomes resistance and is effective in vivo}

Iboxamycin (OPP-3) exhibits markedly improved antibacterial activity when compared to clindamycin and in side-by-side comparisons with azithromycin, vancomycin, doxycycline, levofloxacin, and linezolid against a broad panel of different clinical isolates (Fig. 2, Supplementary Table 1). Broth culture susceptibility analysis revealed that iboxamycin displays 
an extended spectrum of activity against common bacterial pathogens, including those constitutively expressing erm genes that confer resistance to $\mathrm{MLS}_{\mathrm{B}}$ antibiotics (Fig. 2a). For instance, in streptococci, iboxamycin overcame c-ermB-mediated resistance, an increasingly widespread phenotype ${ }^{39,40}$, with MICs $\geq 1,000 \times$ lower than that of clindamycin against $S$. pneumoniae and S. pyogenes strains. Iboxamycin was highly effective against a panel of clinically derived strains of methicillin-resistant Staphylococcus aureus (MRSA, Fig. 2b). Enterococcal pathogens, responsible for widespread and life-threatening healthcare-associated infections ${ }^{41}$ and historically invulnerable to lincosamides, appear broadly susceptible as well: iboxamycin displayed MICs $\leq 2 \mu \mathrm{g} / \mathrm{mL}$ against a panel of Enterococcus faecium and Enterococcus faecalis strains with varying resistance to vancomycin, linezolid, azithromycin, ciprofloxacin, levofloxacin, gentamicin, and doxycycline (Fig. 2b). Notably, iboxamycin exhibited potent activity against $C$. difficile (a CDC-listed Urgent Threat) ${ }^{23}$, which suggests that it may carry reduced risk of promoting C. difficile colitis, but validation of this hypothesis requires further study. Iboxamycin showed activity against Gram-negative species as well - a further departure from approved lincosamides - even inhibiting the growth of a clinically derived strain of Escherichia coli with acquired resistance (c-ermB, MIC $8 \mu \mathrm{g} / \mathrm{mL}$, Fig. 2a). It is interesting that this expanded spectrum of activity relative to clindamycin is not readily explained by traditional physicochemical predictors of Gramnegative activity such as rotatable-bond count, molecular weight, relative polar surface area, or lipophilicity ${ }^{42}$. Broth culture profiling revealed that, like clindamycin, iboxamycin is bacteriostatic but exhibits prolonged effects on bacterial growth following even brief exposure; concentrationdependent cidality was observed for certain highly susceptible strains (Extended Data Fig. 3). 
a

\begin{tabular}{|c|c|c|c|c|}
\hline Species & Description & OPP-3 & Clinda & Azithro \\
\hline S. aureus & ATCC 29213 & 0.06 & 0.125 & 1 \\
\hline S. aureus & ATCC BAA-977, i-ermA & 0.06 & 0.125 & $>64$ \\
\hline S. aureus & ATCC BAA-1707, MRSA & 0.06 & 0.125 & 1 \\
\hline S. aureus & ATCC 700699 & 2 & $>128$ & $>128$ \\
\hline S. aureus & MMX-3035, c-ermA & 4 & $>256$ & $>64$ \\
\hline S. aureus & Clinical, ermA & 1 & $>256$ & $>256$ \\
\hline S. aureus & Clinical, cfr & 2 & $>128$ & $>128$ \\
\hline S. epidermidis & Clinical, cfr & 8 & $>128$ & 32 \\
\hline S. pneumoniae & ATCC 49619, PIRSP & 0.015 & 0.06 & 0.125 \\
\hline S. pneumoniae & ATCC 6303 & 0.03 & 0.06 & 0.25 \\
\hline S. pneumoniae & MMX-3028, c-ermB & 0.25 & 256 & $>64$ \\
\hline S. pyogenes & ATCC 19615 & 0.03 & 0.06 & 0.06 \\
\hline S. pyogenes & MMX-946, c-ermB & 0.25 & $>256$ & $>64$ \\
\hline E. faecalis & ATCC 29212 & 0.06 & 16 & 8 \\
\hline C. difficile & ATCC 700057 & 0.25 & 8 & NT \\
\hline B. fragilis & ATCC 25285 & 0.5 & 1 & NT \\
\hline E. coli & ATCC 25922 & 8 & $>128$ & 4 \\
\hline E. coli & Clinical & 8 & $>128$ & 64 \\
\hline E. coli & Clinical, c-ermB & 8 & $>128$ & 128 \\
\hline E. coli & Clinical, armA & 8 & $>256$ & 4 \\
\hline K. pneumoniae & ATCC 10031 & 0.25 & 8 & 2 \\
\hline K. pneumoniae & Clinical, KPC & 32 & 256 & $>64$ \\
\hline K. pneumoniae & Clinical, FQ-R & 8 & $>128$ & 32 \\
\hline K. oxytoca & Clinical & 8 & $>128$ & 16 \\
\hline A. baumanii & ATCC 19606 & 4 & $>128$ & 64 \\
\hline$P$. aeruginosa & ATCC 27853 & 128 & $>128$ & $>64$ \\
\hline H. influenzae & ATCC 49247 & 1 & 8 & 2 \\
\hline
\end{tabular}

b

\begin{tabular}{|c|c|c|c|c|c|c|c|c|}
\hline Species & Description & OPP-3 & Clinda & Azithro & Vanco & Doxy & Levo & Linez \\
\hline \multirow{6}{*}{ S. aureus } & Clinical, MRSA & 0.06 & 0.25 & 1 & 2 & $\overline{64}$ & 1 & 2 \\
\hline & Clinical, $m s r(A)$ & 0.06 & 0.125 & $>64$ & 1 & 8 & 1 & 4 \\
\hline & Clinical, VISA & 1 & $>64$ & $>64$ & 8 & 8 & 8 & 2 \\
\hline & Clinical, hVISA & 1 & $>64$ & $>64$ & 2 & 16 & 8 & 2 \\
\hline & Clinical, MDR & 1 & $>256$ & $>256$ & 1 & 2 & 32 & 1 \\
\hline & Clinical & 2 & $>64$ & $>64$ & 0.5 & 4 & 8 & 4 \\
\hline \multirow{6}{*}{ S. pneumoniae } & Clinical, c-mefA & 0.03 & 0.06 & 16 & 0.5 & 0.125 & 0.5 & 1 \\
\hline & Clinical & 0.125 & 0.25 & 2 & 1 & 0.25 & 0.25 & 4 \\
\hline & ATCC BAA-334 & 0.125 & 0.25 & 1 & 0.5 & 0.25 & 0.5 & 4 \\
\hline & ATCC BAA-255 & 0.125 & 0.25 & 2 & 1 & 0.25 & 0.25 & 4 \\
\hline & Clinical & 0.25 & $>64$ & $>64$ & 2 & 2 & 0.25 & 4 \\
\hline & ATCC 700673 & 0.5 & $>64$ & $>64$ & 0.5 & 4 & 0.5 & 4 \\
\hline \multirow{9}{*}{ E. faecalis } & Clinical & $\leq 0.06$ & 16 & 4 & 1 & 0.25 & 1 & 4 \\
\hline & Clinical, Line-R & $\leq 0.06$ & 8 & 4 & 2 & 64 & 8 & 8 \\
\hline & Clinical, MDR & 0.125 & 128 & $>256$ & $>256$ & 16 & 64 & 8 \\
\hline & Clinical & 1 & $>128$ & $>64$ & 8 & 0.125 & 2 & 2 \\
\hline & Clinical & 1 & $>256$ & $>128$ & 16 & 0.5 & 64 & 2 \\
\hline & Clinical, VRE & 1 & $>256$ & $>128$ & 32 & 0.5 & 8 & 2 \\
\hline & Clinical, c-ermB & 1 & $>256$ & $>64$ & $>64$ & 16 & $>64$ & 2 \\
\hline & Clinical, VRE & 2 & $>256$ & $>128$ & $>128$ & 1 & 64 & 16 \\
\hline & Clinical, VRE & 2 & $>256$ & $>128$ & 32 & 8 & 32 & 4 \\
\hline \multirow{6}{*}{ E. faecium } & Clinical, MDR & $\leq 0.06$ & $>64$ & 8 & $>256$ & 16 & 128 & 64 \\
\hline & Clinical, VRE & 0.25 & $>128$ & $>128$ & $>128$ & 2 & 4 & 2 \\
\hline & Clinical, VRE & 1 & $>64$ & $>64$ & $>64$ & 4 & $>64$ & 4 \\
\hline & Clinical, VRE & 1 & $>256$ & $>128$ & $>128$ & 0.25 & 128 & 4 \\
\hline & Clinical, VRE & 1 & $>256$ & $>128$ & $>128$ & 0.25 & 128 & 4 \\
\hline & Clinical, VRE & 1 & $>256$ & $>128$ & $>128$ & 0.25 & $>128$ & 4 \\
\hline
\end{tabular}

C

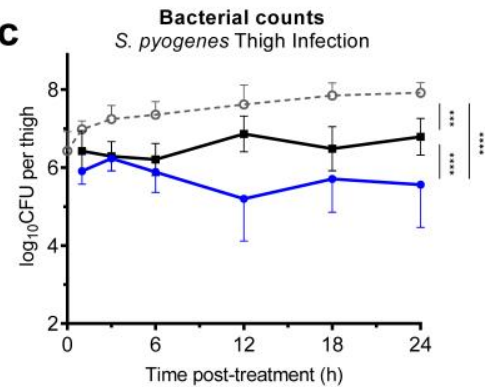

d

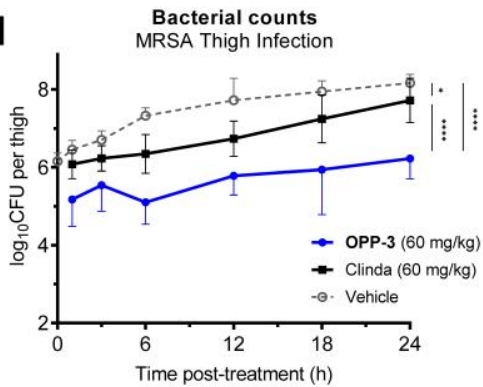

e

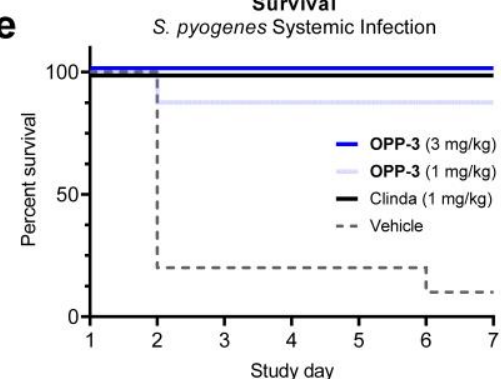

Figure 2 | In vitro and in vivo antibacterial activity of iboxamycin (OPP-3). a, MICs $(\mu \mathrm{g} / \mathrm{mL})$ of iboxamycin and clindamycin against standard and multidrug-resistant Gram-positive and Gram-negative strains. b, MICs of antibiotics evaluated against clinically derived strains of $S$. aureus, E. faecium, and E. faecalis displaying a range of multidrug resistance phenotypes. $\mathbf{c}, \mathbf{d}$, Pharmacodynamic effects of iboxamycin in a murine neutropenic thigh infection model employing S. pyogenes ATCC 19615 or S. aureus (MRSA). Points represent mean values across two experiments, and error bars depict standard deviation from the mean ( $\mathrm{n}=16$ counts across 4 independent animals per time point). Statistical comparison of 24-h time points was conducted using one-way ANOVA with Tukey's multiple comparison test. $* p<0.05, * * * p<0.0005, * * * * p<0.0001$. e, Survival over time of mice infected with $S$. pyogenes ATCC 19615 treated with iboxamycin $(n=8)$, clindamycin $(n=8)$, or vehicle $(n=10)$. Clinda, clindamycin; Azithro, azithromycin; Vanco, vancomycin; Doxy, doxycycline; Levo, levofloxacin; Linez, linezolid; NT, not tested; iErmA/B, inducible erythromycin ribosome methyltransferase A; c-erm $A / B$, constitutively expressed erythromycin methyltransferase A/B gene; MRSA, methicillin-resistant Staphylococcus aureus; frr, chloramphenicol-florfenicol resistance gene; PIRSP, penicillin-intermediate resistant Streptococcus pneumoniae; armA, aminoglycoside resistance methylase gene; KPC, Klebsiella pneumoniae carbapenemase; FQ-R, fluoroquinolone-resistant; $m s r(A)$, erythromycin resistance ATPbinding protein MsrA gene; VISA, vancomycin-intermediate resistant Staphylococcus aureus; hVISA, vancomycinheteroresistant Staphylococcus aureus; MDR, multidrug-resistant; c-mefA, constitutively expressed macrolide efflux protein A gene; VRE, vancomycin-resistant Enterococcus; Line-R, linezolid-resistant.

To assess the efficacy of oxepanoprolinamide antibiotics, we evaluated iboxamycin (OPP-3) in animal models of infection. In cell-culture safety profiling, iboxamycin was non-hemolytic and 
non-toxic toward mammalian cells $\left(\mathrm{GI}_{50}>50 \mu \mathrm{M}\right.$, Extended Data Fig. 4a,d-f), and had no effect on membrane integrity or mitochondrial function (Extended Data Fig. 4b,c) ${ }^{43,44}$. In mice, iboxamycin achieved greater exposure than clindamycin, exhibiting an intravenous mean residence time (MRT) of $1.2 \mathrm{~h}$ and $24 \%$ oral bioavailability (Extended Data Table 1). With these data in hand, we next evaluated the pharmacodynamic effects of iboxamycin in a murine neutropenic thigh-infection model using S. pyogenes ATCC 19615 and S. aureus (MRSA) as test organisms. In both experiments, iboxamycin achieved statistically significant reductions in bacterial burden $24 \mathrm{~h}$ following an intraperitoneal dose, when compared to clindamycin- and vehicle-treated controls (Fig. 2c, d). The in vivo efficacy of iboxamycin was also studied in a murine model of systemic infection, in which mice received an intraperitoneal dose of $S$. pyogenes ATCC 19615, followed by intravenous administrations of iboxamycin (1, 3, or $10 \mathrm{mg} / \mathrm{kg}$ ), clindamycin phosphate $(1,3$, or $10 \mathrm{mg} / \mathrm{kg})$, or vehicle twice daily for two days. Treatment with iboxamycin was well-tolerated at all dose levels in this experiment, and resulted in complete rescue of infected animals, with all mice treated at the 3 and $10 \mathrm{mg} / \mathrm{kg}$ dose levels surviving (Fig. 2e, Extended Data Table 3).

\section{Iboxamycin targets wild-type and drug-resistant ribosomes}

The encouraging profile of iboxamycin (OPP-3) prompted us to investigate its on-target activity and the structural basis for its improved antibacterial properties. While spontaneous resistance was not observed in standard strains of $S$. aureus or E. faecalis over the course of three-day experiments (see Methods), we successfully selected iboxamycin-resistant mutants using E. coli SQ110DTC, a strain specifically designed for selecting mutations in rRNA that render cells resistant to ribosome-targeting inhibitors $^{45}$. This strain lacks six of seven rRNA alleles and the major multidrug efflux pump TolC. Upon plating SQ110DTC cells on an agar plate containing iboxamycin, resistant clones with MICs in the range of $2-4 \mu \mathrm{g} / \mathrm{mL}$ appeared with a frequency of $\sim 10^{-8}$. Of the 14 randomly selected clones that were sequenced, all carried the single-nucleotide mutations A2058G or A2059G within the 23S rRNA (Extended Data Table 2), corresponding to changes in the canonical lincosamide binding site. This result demonstrated that, in analogy to lincosamides, iboxamycin targets the bacterial ribosome.

In order to understand the effect of iboxamycin (OPP-3) on ribosomal function, we performed primer extension inhibition analysis ("toeprinting"), an in vitro technique that detects the position 
of the drug-arrested ribosome on mRNA in cell-free translation. ${ }^{46,47}$. Because the ribosome is exposed to the antibiotic prior to the initiation of translation in this assay, a strong inhibitor of peptide-bond formation should arrest the ribosome at the start codons of open reading frames (ORFs), whereas a more weakly binding inhibitor of the catalytic center would be expected to afford a degree of escape from start codons. In our toeprinting experiment, consistent with prior reports $^{45}$, clindamycin arrested translation at the start codons of the model ORFs, yet allowed a fraction of ribosomes to translate ORFs up to the trap codon, at which point ribosomes were arrested due to the lack of isoleucyl-tRNA in the in vitro translation system (Fig. 3). In contrast, present at the same concentration $(50 \mu \mathrm{M})$, iboxamycin firmly locked the ribosome at the start codons, allowing virtually no escape, suggesting that iboxamycin associates more strongly with the ribosome than does clindamycin.
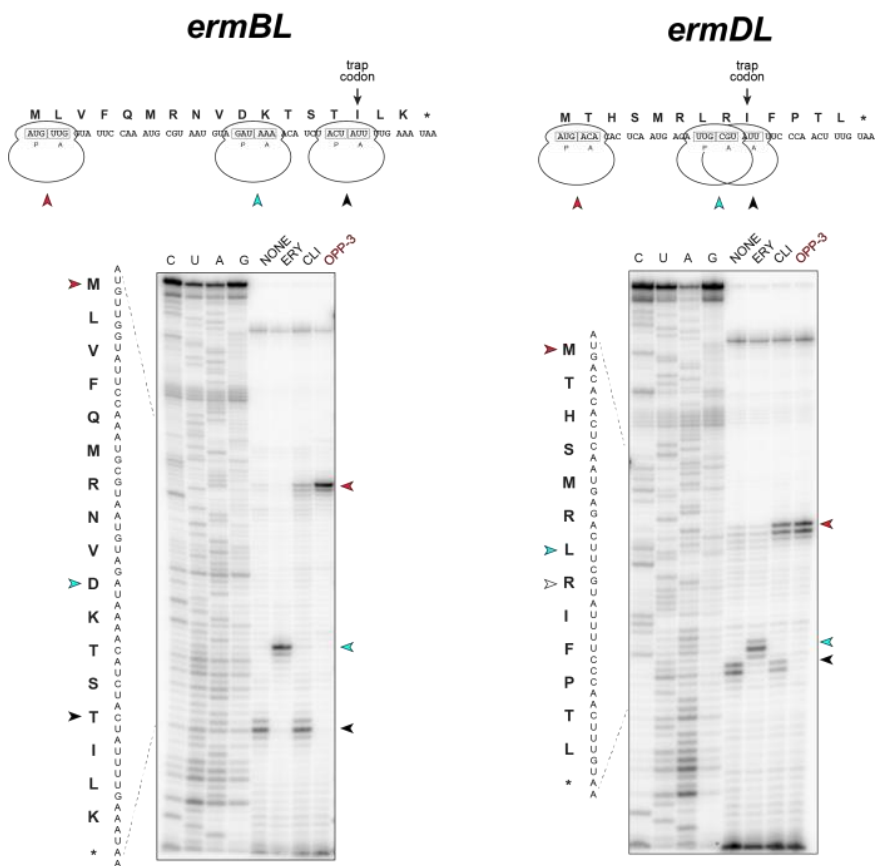

Figure 3 | Iboxamycin (OPP-3) efficiently arrests translation at the start codon. Toeprinting analysis of sites of iboxamycin-induced translation arrest of ErmBL and ErmDL leader peptides. Because all reactions contained mupirocin, an inhibitor of Ile-tRNA synthetase, the ribosomes that escape inhibition by ribosome-targeting antibiotics are trapped at the codon preceding Ile (black arrowheads). Red arrowheads mark translation arrest at the start codon, while cyan arrowheads denote known erythromycin-induced arrest sites D10 (ermBL) and L7 (ermDL). ERY, erythromycin; CLI, clindamycin.

To illuminate the structural basis for this improved activity, we determined the structure of iboxamycin (OPP-3) bound to the bacterial ribosome by X-ray crystallography. 70S Ribosomes 
from the Gram negative bacterium Thermus thermophilus (Tth) were co-crystallized with iboxamycin, mRNA, non-hydrolyzable aminoacyl-tRNA analog fMet-NH-tRNA ${ }_{i}{ }^{\text {Met }}$ (located

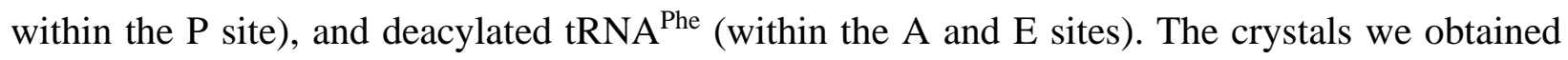
diffracted to 2.50- $\AA$ resolution (Supplementary Table 2). The unbiased $F_{o}-F_{c}$ difference Fourier map revealed positive electron density peaks resembling characteristic chemical features of iboxamycin (Fig. 4a), and confirmed that the antibiotic binds in the canonical binding pocket within the large ribosomal subunit (Fig. 4b), spanning the peptidyl transferase center (PTC) and extending into the nascent peptide exit tunnel (NPET) (Supplementary Movie 1). 

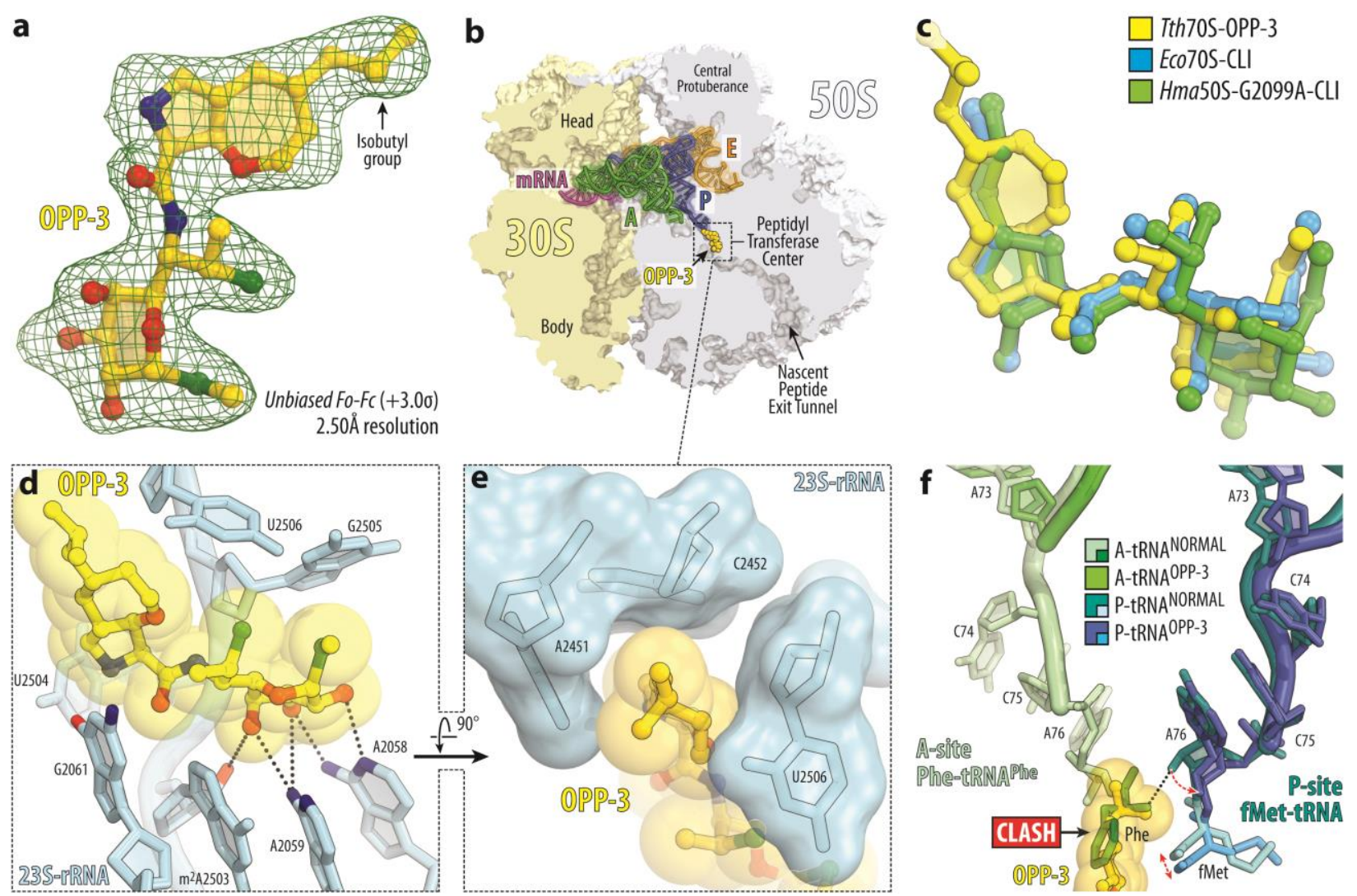

Figure 4 | Structure of iboxamycin (OPP-3) in complex with the 70S ribosome, mRNA, and tRNAs. a, Unbiased $F_{o}-F_{c}$ electron density map of iboxamycin in complex with the Tth $70 \mathrm{~S}$ ribosome (green mesh) contoured at $3.0 \sigma . \mathbf{b}$, Overview of the drug-binding site (yellow) in the Tth $70 \mathrm{~S}$ ribosome viewed as a cross-cut section through the nascent peptide exit tunnel. The 30S subunit is shown in light yellow, the 50S subunit is in light blue, the mRNA is in magenta, and the A-, P-, and E-site tRNAs are colored green, dark blue, and orange, respectively. c, Superposition of the ribosome-bound oxepanoprolinamide iboxamycin (yellow) with the previous structures of clindamycin bound to the tRNA-free $70 \mathrm{~S}$ ribosome from eubacterium E. coli (blue, $\mathrm{PDB}$ entry $4 \mathrm{~V} \mathrm{~V}^{32}$ ) or to $50 \mathrm{~S}$ ribosomal subunit from archaeon $H$. marismortui harboring G2099A mutation in its 23S rRNA (green, PDB entry $1 \mathrm{YJN}^{48}$ ). All structures were aligned based on domain $\mathrm{V}$ of the 23S rRNA. d, e, Close-up views of iboxamycin bound in the PTC, highlighting $\mathrm{H}$-bond interactions (dashed lines) and the intercalation of the 7'-isobutyl substituent within the A-site cleft formed by A2451 and C2452 of the 23S rRNA. (f) Superimposed models of the A- and P-site tRNAs from the current iboxamycin-ribosome complex structure (green and blue, respectively) with the previous structure of drug-free ribosome containing the same tRNAs (PDB entry $6 \mathrm{XHW}^{52}$ ). Note that the binding of iboxamycin to the ribosome prevents accommodation of the aminoacyl moiety and proper placement of the CCA-end of the incoming A-site tRNA.

The binding of iboxamycin (OPP-3) to the functional Tth 70S ribosome complex containing mRNA and tRNAs corresponds closely to the binding of clindamycin to the tRNA-free 70S ribosome of $E$. coli ${ }^{32}$ or the large ribosomal subunit of the archaeon Haloarcula marismortui (Fig. 4c) $)^{48}$. As with clindamycin and lincomycin, a network of hydrogen bonds appears to anchor the aminooctose moiety of iboxamycin to the NPET nucleotides A2058, A2059, and A2503. Likewise, the cationic aminoacyl residue of iboxamycin occupies a hydrophilic pocket formed by the PTC residues G2061 and U2504 (Fig. 4d), displacing a divalent magnesium ion that is otherwise 
observed in this location in the drug-free ribosome ${ }^{49,50}$. Consistent with our design hypothesis (Extended Data Fig. 1b), the oxepane ring of ribosome-bound iboxamycin overlays closely with the $\mathrm{C}^{\prime}$ and $\mathrm{C}^{\prime}$ ' $n$-propyl-group atoms of clindamycin, favors the $\mathrm{C} \gamma$-endo proline ring pucker, and - most notably - presents the 7'-isobutyl group for interaction with the A-site cleft formed by $23 \mathrm{~S}$ rRNA residues A2451 and C2452 (Fig. 4e). This cleft, which normally accommodates the sidechains of incoming amino acids, plays a key role in the positioning of aminoacylated 3' ends of Asite tRNAs within the PTC during translation ${ }^{49,50}$. It is noteworthy that the electron density corresponding to the CCA terminus of the A-site tRNA is poorly resolved in our structure, suggesting that iboxamycin blocks proper coordination of the 3 '-terminal nucleotides and the aminoacyl residue of the A-site tRNA (Extended Data Fig. 5a). Interestingly, the 7'-isobutyl substituent of iboxamycin extends deep enough within the A-site cleft to overlap not only with incoming amino acids (as the $n$-propyl group of clindamycin does, albeit to a lesser extent) but also with the P-site amino acid (Fig. 4f). This observation, together with the toeprinting results described above, leads us to propose that iboxamycin functions as a specific inhibitor of translation initiation, similar to the pleuromutilin antibiotics ${ }^{51}$.

The extraordinary activity of iboxamycin (OPP-3) against bacteria harboring erm and $c f r$ resistance genes prompted us to determine the crystal structure of the compound bound to the $\mathrm{m}_{2}^{6} \mathrm{~A} 2058$ ribosome as well. Erm-mediated methylation of A2058 confers resistance to clindamycin by blocking the association of its 7-Cl-MTL residue with the NPET, ${ }^{52}$ and because iboxamycin bears the same aminooctose residue as clindamycin, its ability to overcome MLS $_{B}$ resistance was unexpected. Erm-modified ribosomes with A2058 dimethylation levels of 60\% were isolated from Tth cells expressing the erm gene from Bifidobacterium thermophilum ${ }^{52}$. Cocrystallization of these ribosomes with iboxamycin provided crystals diffracting to $2.60-\AA$ resolution (Supplementary Table 2). Remarkably, the corresponding electron-density map revealed that iboxamycin binds to the methylated ribosome in a manner almost identical to its positioning within the wild-type ribosome, while $\mathrm{m}_{2}^{6} \mathrm{~A} 2058$ undergoes a movement of $\sim 2 \AA$ relative to its wild-type position in order to accommodate the antibiotic (Fig. 5). $\mathrm{N}^{6}$-dimethylation, together with this previously unknown displacement of A2058, disrupts the two hydrogen bonds typically formed between this residue and the aminooctose residue of lincosamides; loss of these bonds is apparently sufficient to destabilize binding of clindamycin to the Erm-modified ribosome. However, new hydrophobic interactions between iboxamycin and the A-site cleft appear to 
compensate for the loss of interactions with the modified nucleobase, providing sufficiently high affinity for iboxamycin to overcome resistance. This may suggest a generalizable approach to the design of other antibiotics overcoming similar resistance mechanisms.
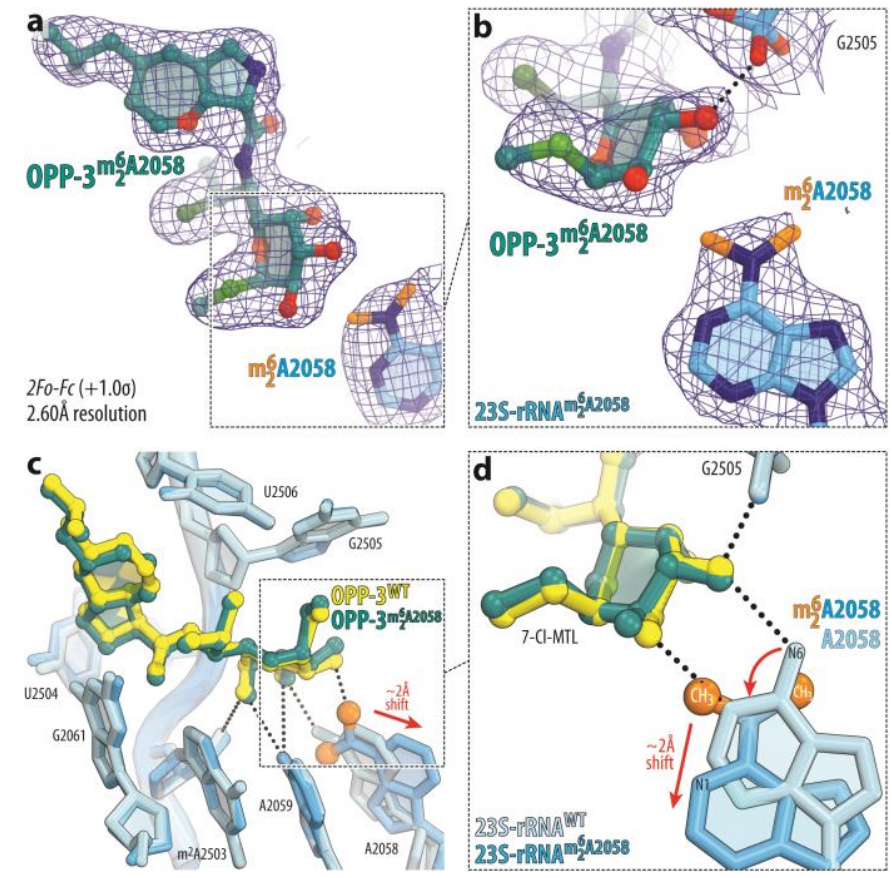

Figure 5 | Structure of iboxamycin (OPP-3) bound to the Erm-methylated 70S ribosome. a, b, Electron density map (blue mesh) contoured at 3.0 $\sigma$ of iboxamycin (teal) in complex with the Erm-modified Tth 70S ribosome containing $\mathrm{N}^{6}$-dimethylated A2058 residue in the $23 \mathrm{~S}$ rRNA. Nitrogen atoms are colored dark blue, oxygen atoms are red, and the methyl groups of $\mathrm{m}_{2}^{6} \mathrm{~A} 2058$ are highlighted in orange. $\mathbf{c}, \mathbf{d}$, Superposition of the iboxamycin structure (yellow) in complex with the WT 70S ribosome, containing unmodified residue A2058, and the structure of iboxamycin (teal) in complex with Erm-modified 70S ribosome, containing $\mathrm{m}_{2}^{6} \mathrm{~A} 2058$. 23S rRNA residues are shown in light blue for the WT ribosome and medium blue for the Erm-modified ribosome. Hydrogen bonds are depicted with dashed lines. Note that the binding positions of iboxamycin are almost identical in the two structures, while iboxamycin binding to the Erm-modified ribosome causes substantial movement of $\mathrm{m}_{2}^{6} \mathrm{~A} 2058$ from its canonical position (red arrow).

\section{Conclusion}

Using a platform for antibiotic discovery rooted in component-based synthesis and structureguided design, we identified the emergent oxepanoprolinamide antibiotic class. Departing radically from their progenitors, the oxepanoprolinamides display exceptional activity against high-priority enterococcal pathogens, overcome $\mathrm{erm}$ - and $\mathrm{cfr}$-mediated multidrug resistance, and display moderate activity against Gram-negative pathogens. Methodologically, our findings attest to the role of empiricism in antibiotics discovery, as the activity of oxepanoprolinamides against posttranscriptionally modified ribosomes could not have been predicted on the basis of prior 
structural knowledge. Further refinement of the scaffold, including modifications to the aminooctose residue whose unexpected capacity to displace $\mathrm{m}_{2}^{6} \mathrm{~A} 2058$ we illustrate here, is likely to expand the oxepanoprolinamides' spectrum of activity further. These findings, together with the safety, pharmacokinetic profile, and efficacy of iboxamycin in mice, reaffirm the capacity for chemical synthesis to replenish our armamentarium, delivering antibiotics capable of overcoming dangerous resistance threats. 


\section{AUTHOR CONTRIBUTIONS}

M.J.M. identified the oxepanoprolinamide scaffold and the synthetic routes used to prepare analogs OPP-1, OPP-2, OPP-4, and OPP-5. A.P. designed and performed MIC, TK, PAE, PASME, and animal-infection experiments. E.A.S. and Y.S.P. designed and performed X-ray crystallography experiments. K.J.S. identified 7'-alkyl oxepanoprolinamides and the synthetic route used to prepare analogs OPP-6, epi-OPP-3, and iboxamycin (OPP-3) from ketone 10. D.K. and A.S.M. selected and sequenced iboxamycin-resistant SQ110DTC clones and conducted ribosome toe-printing analysis. M.J.M., K.J.S., J.D.M., D.W.T., G.T., A.R.P., and K.J.Y.W. synthesized and characterized oxepanoprolinamide analogs. A.P. and R.P.L. performed mammalian cell experiments. A.P. and K.C. designed and performed the pharmacokinetic experiments. A.G.M., A.S.M., and Y.S.P. supervised the experiments. All authors interpreted the results. M.J.M, A.G.M, A.S.M, and Y.S.P. wrote the manuscript. All authors discussed the results and commented on the manuscript.

\section{ACKNOWLEDGMENTS}

The authors thank Chih-Wei Chen for help with initial X-ray diffraction data collection, and Maxim Svetlov for providing Erm-methylated ribosomes used in our structural studies. We gratefully acknowledge David R. Andes, Michael S. Gilmore, François H. Lebreton, Thomas J. Dougherty, David P. Nicolau, Patrice Courvalin, Catherine Grillot-Courvalin, Heinz E. Moser, Su Chiang, Barry Wohl, Curtis Keith, Ruchia Duggal, and Karin M. Otte for their invaluable input in the course of our research. The staff at NE-CAT beamlines 24ID-C and 24ID-E are gratefully acknowledged for their help with data collection, especially Drs. Malcolm Capel, Frank Murphy, Igor Kourinov, Anthony Lynch, Surajit Banerjee, David Neau, Jonathan Schuermann, Narayanasami Sukumar, James Withrow, Kay Perry, and Cyndi Salbego. We thank Shao-Liang Zheng for X-ray crystallographic data collection and structure determination of compounds 6 and OPP-3. We thank Dean L. Shinabarger and Chris Pillar of Micromyx, L.L.C., William J. Weiss of the UNT Health Science Center, and Michael D. Cameron of TSRI Florida for in vitro profiling of compounds. Ligand Pharmaceuticals kindly provided the Captisol used in our animal experiments. M.J.M. was supported by the National Science Foundation Graduate Research Fellowship under Grant No. DGE1144152. K.J.Y.W. was supported by a National Science Scholarship (Ph.D.) by the Agency for Science, Technology and Research, Singapore. G.T. 
received postdoctoral support from the Deutsche Forschungsgemeinschaft (TE1311-1-1). This work is based upon research conducted at the Northeastern Collaborative Access Team (NE-CAT) beamlines, which are funded by the National Institute of General Medical Sciences from the National Institutes of Health (P30-GM124165). The Eiger 16M detector on 24ID-E beamline is funded by a NIH-ORIP HEI grant (S10-OD021527 to NE-CAT) This research used resources of the Advanced Photon Source, a U.S. Department of Energy (DOE) Office of Science User Facility operated for the DOE Office of Science by Argonne National Laboratory under Contract No. DEAC02-06CH11357. Extraordinary facility operations were supported in part by the DOE Office of Science through the National Virtual Biotechnology Laboratory, a consortium of DOE national laboratories focused on the response to COVID-19, with funding provided by the Coronavirus CARES Act. This work was supported by Illinois State startup funds to Y.S.P. and National Institutes of Health R21-AI137584 (to A.S.M. and Y.S.P.), R01-GM132302 (to Y.S.P), and R35GM127134 (to A.S.M.). A.G.M. gratefully acknowledges support from the LEO Foundation (grant LF18006) and the Blavatnik Biomedical Accelerator at Harvard University.

\section{ACCESSION NUMBERS}

Coordinates and structure factors will be deposited in the RCSB Protein Data Bank for the T. thermophilus $70 \mathrm{~S}$ ribosome in complex with iboxamycin, mRNA, deacylated A-site tRNA ${ }^{\text {Phe }}$, aminoacylated P-site fMet-NH-tRNA ${ }_{i}^{\text {Met }}$, and deacylated E-site tRNA ${ }^{\text {Phe }}$; and for the $\mathrm{m}_{2}^{6} \mathrm{~A} 2058$ T. thermophilus $70 \mathrm{~S}$ ribosome in complex with iboxamycin, mRNA, deacylated A-site tRNA ${ }^{\text {Phe }}$, aminoacylated P-site fMet-NH-tRNA ${ }_{i}{ }^{\text {Met }}$. Single-crystal X-ray crystallographic data for compound 6 have been deposited at the Cambridge Crystallographic Data Centre under deposition number 2072277.

\section{CONFLICT OF INTEREST STATEMENT}

A.G.M., M.J.M., K.J.S., J.D.M., and G.T are inventors in a provisional patent application submitted by the President and Fellows of Harvard College covering antibiotics of the type described in this work. A.G.M., M.J.M., and K.J.S. have filed an international patent application WO/2019/032936 'Lincosamide Antibiotics and Uses Thereof'. A.G.M. and M.J.M. have filed an international patent application WO/2019/032956 'Lincosamide Antibiotics and Uses Thereof'. 


\section{EXTENDED DATA DISPLAY ITEMS}
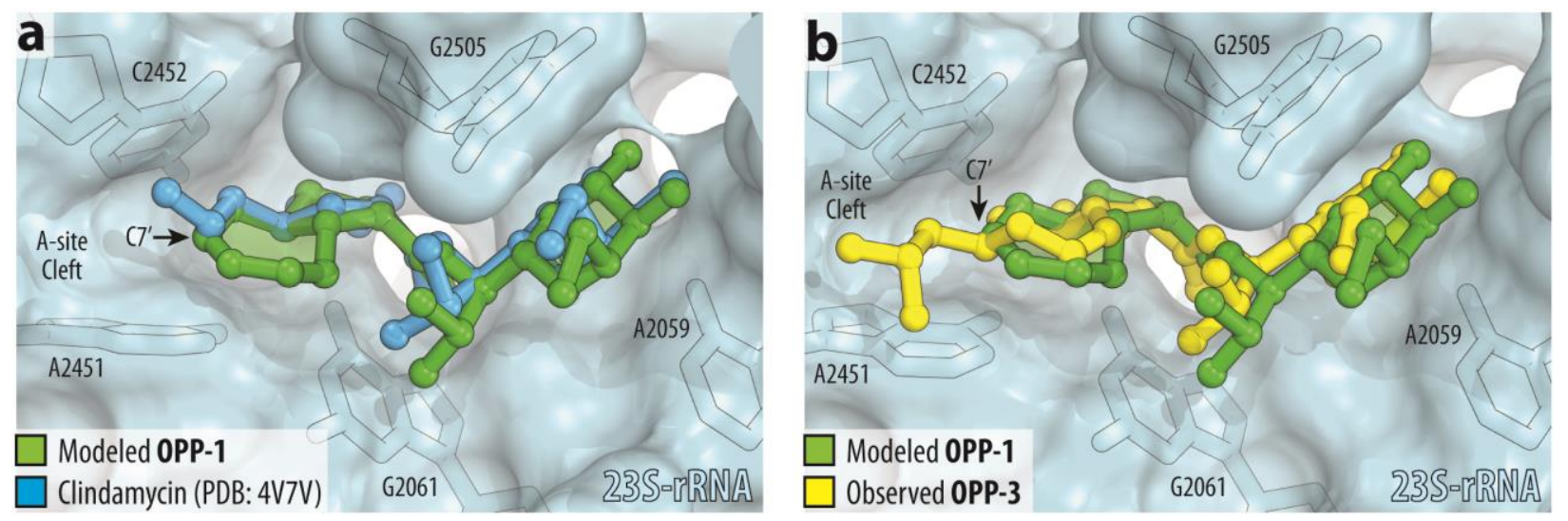

Extended Data Figure 1 | Structure-based design of 7'-substituted oxepanoprolinamides. a,

Superposition of the X-ray crystal structure of ribosome-bound lincosamide antibiotic clindamycin (2, blue, PDB entry $4 \mathrm{~V} 7 \mathrm{~V}^{32}$ ) with the energy-minimized structure of OPP-1 (green). Note that in this configuration, the C7'-atom of OPP-1 contacts the lipophilic surface of the A-site cleft. b, The same structure, overlaid with the X-ray crystal structure of iboxamycin (OPP-3, yellow) bound to the bacterial ribosome. 


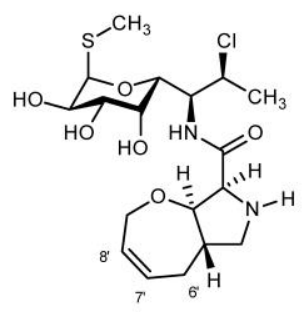

OPP-1

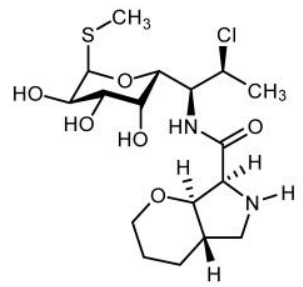

OPP-5<smiles>CSC1C(O)C(O)C(C(NC(=O)[C@@H]2NC[C@@H]3C[C@H](F)CCO[C@H]32)C(C)Cl)C(O)C1O</smiles>

OPP-2<smiles>CSC1C(O)C(O)C(C(NC=O)C(C)Cl)C1O</smiles><smiles>CC(=O)[C@@H]1NC[C@H]2CNC(CC(C)C)=CCO[C@@H]21</smiles><smiles>CSC1C(O)C(O)C(O)C1C(NC(=O)[C@@H]1NC[C@@H]2CCCCO[C@H]21)C(C)Cl</smiles>

OPP -4<smiles>CSC1C(O)C(O)C(C(NC=O)C(C)Cl)C1O</smiles><smiles>C=C(C)[C@H]1NC[C@H]2C[C@@H](CC(C)C)CCO[C@H]2[C@H]1C(C)=O</smiles>

\begin{tabular}{|c|c|c|c|c|c|c|c|c|c|}
\hline & Species & Description & Clinda & OPP-1 & OPP-2 & OPP-4 & OPP-5 & OPP-6 & epi-OPP-3 \\
\hline \multirow{6}{*}{ 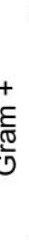 } & S. aureus & ATCC 29213 & 0.125 & 0.125 & 0.25 & 0.125 & 0.5 & 0.06 & $\leq 0.06$ \\
\hline & S. pneumoniae & ATCC 49619 & 0.06 & $\leq 0.06$ & $\leq 0.06$ & $\leq 0.03$ & 0.06 & $\leq 0.03$ & $\leq 0.06$ \\
\hline & S. pneumoniae & MMX 3028; c-ermB & 256 & 64 & 64 & NT & NT & 8 & 0.5 \\
\hline & S. pyogenes & ATCC 19615 & 0.06 & $\leq 0.03$ & $\leq 0.03$ & $\leq 0.03$ & $\leq 0.03$ & $\leq 0.03$ & $\leq 0.06$ \\
\hline & S. pyogenes & MMX 946; c-ermB & $>256$ & 16 & 4 & NT & NT & 32 & 0.5 \\
\hline & E. faecalis & ATCC 29212 & 16 & 16 & 4 & $>32$ & 32 & 0.125 & $\leq 0.06$ \\
\hline & K. pneumoniae & ATCC 10031 & 8 & $>64$ & 16 & 8 & $>32$ & NT & NT \\
\hline & E. coli & ATCC 25922 & $>128$ & $>64$ & $>64$ & 32 & $>32$ & 32 & 16 \\
\hline & A. baumanii & ATCC 19606 & $>128$ & $>32$ & $>32$ & $>32$ & $>32$ & NT & NT \\
\hline & H. influenzae & ATCC 49247 & 8 & 1 & 4 & 8 & 32 & 8 & 2 \\
\hline
\end{tabular}

Extended Data Figure 2 | MICs $(\mu \mathrm{g} / \mathrm{mL})$ of antibiotics containing a bicyclic aminoacyl residue. Selected analogs illustrate the effects of $7^{\prime}$ substitution, ring size, and saturation on antibacterial activity. Clinda, clindamycin; c-ermB, constitutively expressed erythromycin ribosome methylase B gene. 
a

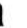
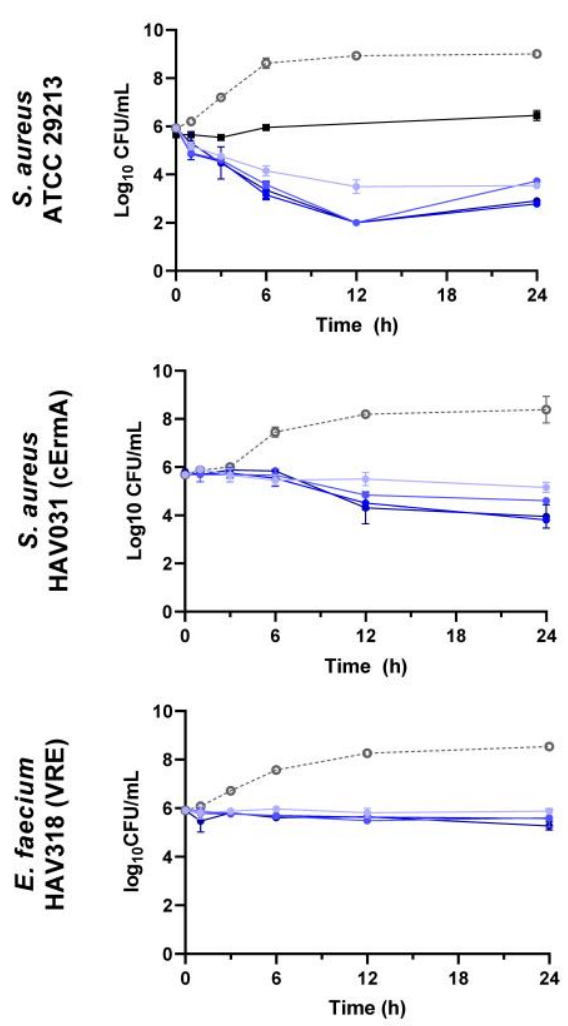

Post-Antibiotic Effect
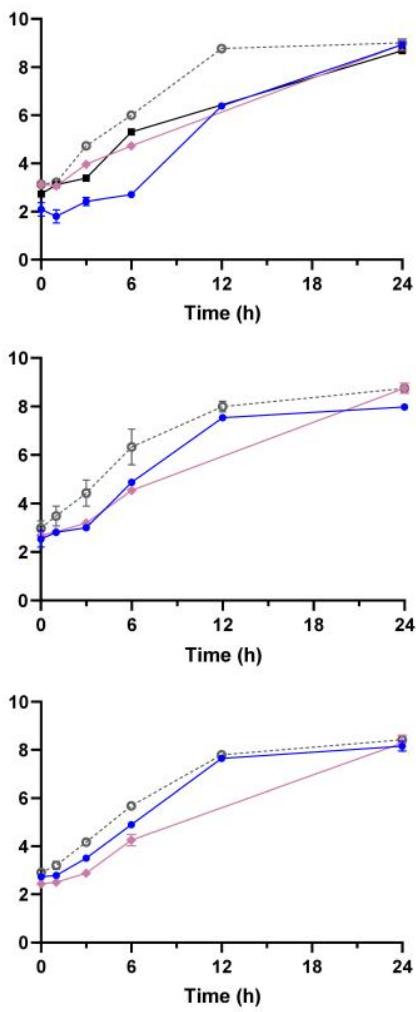

Post-Antibiotic Sub-MIC Effect
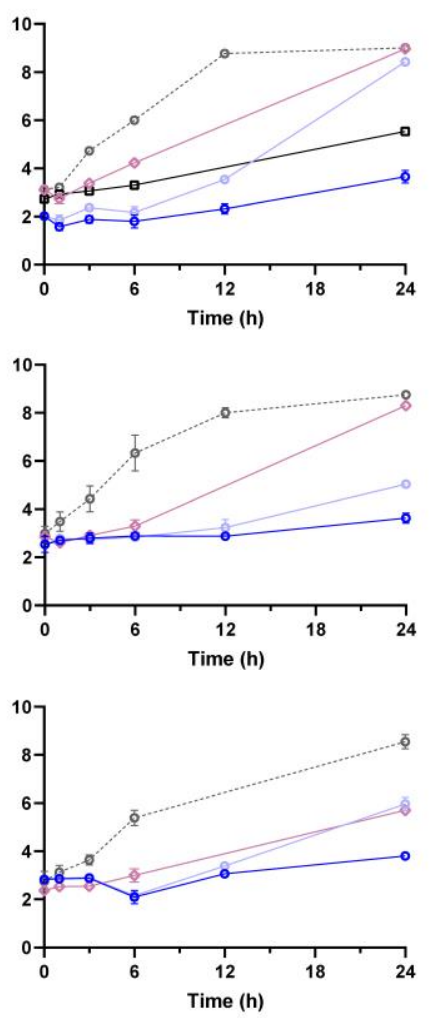

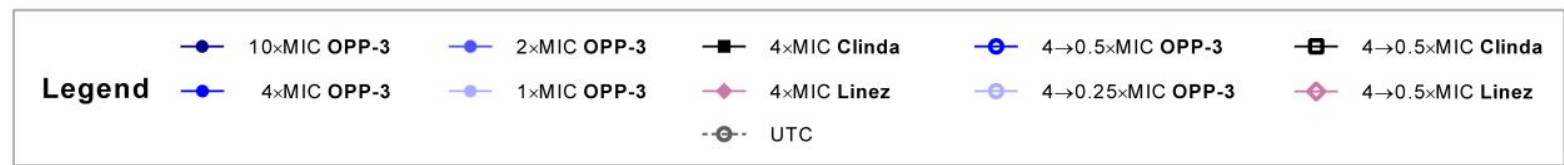

b

\begin{tabular}{|c|c|c|c|c|c|c|c|c|c|c|c|c|c|c|}
\hline \multirow{3}{*}{ Strain } & \multirow{3}{*}{ Treatment } & \multicolumn{4}{|c|}{ Post-Antibiotic Effect (h) } & \multirow{3}{*}{$\begin{array}{r}\text { Pre-treatment } \\
\text { Treatment }\end{array}$} & \multicolumn{8}{|c|}{ Post-Antibiotic Sub-MIC Effect (h) } \\
\hline & & \multirow{2}{*}{$1 \times \mathrm{MIC}$} & \multirow{2}{*}{$2 \times \mathrm{MIC}$} & \multirow{2}{*}{$4 \times \mathrm{MIC}$} & \multirow{2}{*}{$10 \times \mathrm{MIC}$} & & \multicolumn{2}{|c|}{$1 \times \mathrm{MIC}$} & \multicolumn{2}{|c|}{$2 \times \mathrm{MIC}$} & \multicolumn{2}{|c|}{$4 \times \mathrm{MIC}$} & \multicolumn{2}{|c|}{$10 \times \mathrm{MIC}$} \\
\hline & & & & & & & $1 / 4 \mathrm{MIC}$ & $1 / 2 \mathrm{MIC}$ & $1 / 4 \mathrm{MIC}$ & $1 / 2 \mathrm{MIC}$ & $1 / 4 \mathrm{MIC}$ & $1 / 2 \mathrm{MIC}$ & $1 / 4 \mathrm{MIC}$ & $1 / 2 \mathrm{MIC}$ \\
\hline \multirow{3}{*}{ S. aureus ATCC 29213} & Linezolid & 1.5 & 2.3 & 1.8 & 2.8 & & 3.3 & 5.3 & 4.3 & 7.5 & 3.5 & 6.8 & 2.8 & 5.8 \\
\hline & Clindamycin & 1.5 & 1.5 & 1.6 & 2.1 & & 4.7 & 10.1 & 4.9 & 10.0 & 5.3 & 9.2 & 5.1 & 8.0 \\
\hline & OPP-3 & 2.1 & 4.2 & 4.6 & 5.2 & & 6.2 & 13.8 & 8.0 & 15.6 & 7.9 & 17.3 & 7.4 & $>24$ \\
\hline \multirow{2}{*}{ S. aureus HAV031 (cErmA) } & Linezolid & 2.1 & 2.2 & 2.4 & 2.2 & & 5.0 & 9.7 & 4.5 & 9.3 & 6.2 & 9.9 & 5.5 & 9.9 \\
\hline & OPP-3 & 2.3 & 2.4 & 2.3 & 5.1 & & 12.8 & 13.3 & 13.7 & $>24$ & 12.4 & 21.4 & 12.7 & $>24$ \\
\hline \multirow{2}{*}{ E. faecium HAV318 (VRE) } & Linezolid & 0.8 & 1.1 & 1.4 & 4.4 & & 8.9 & 13.4 & 8.7 & 19.9 & 6.9 & 17.4 & 10.5 & $>24$ \\
\hline & OPP-3 & 1.8 & 1.9 & 1.7 & 1.8 & & 9.7 & 19.9 & 10.5 & 23.0 & 11.3 & 21.5 & 11.4 & 21.7 \\
\hline
\end{tabular}

Extended Data Figure 3 | Time-kill kinetics, post-antibiotic effect, and post-antibiotic subMIC effect data of iboxamycin (OPP-3) against susceptible strains. a, Arrayed growth curves for three susceptible strains showing concentration effects on growth inhibition (time-kill), growth kinetics following exposure to antibiotic at $4 \times \mathrm{MIC}(\mathrm{PAE})$, and growth kinetics under sub-MIC concentrations following exposure to antibiotic at $4 \times$ MIC (PA-SME). Points represent the mean of bacterial counts ( $n=2)$, while error bars depict standard deviations from the mean. $\mathbf{b}$, Tabulated 
PAE and PA-SME durations (determined as the difference in time required for bacterial counts to rise $10 \times$ between experimental and untreated control arms). 

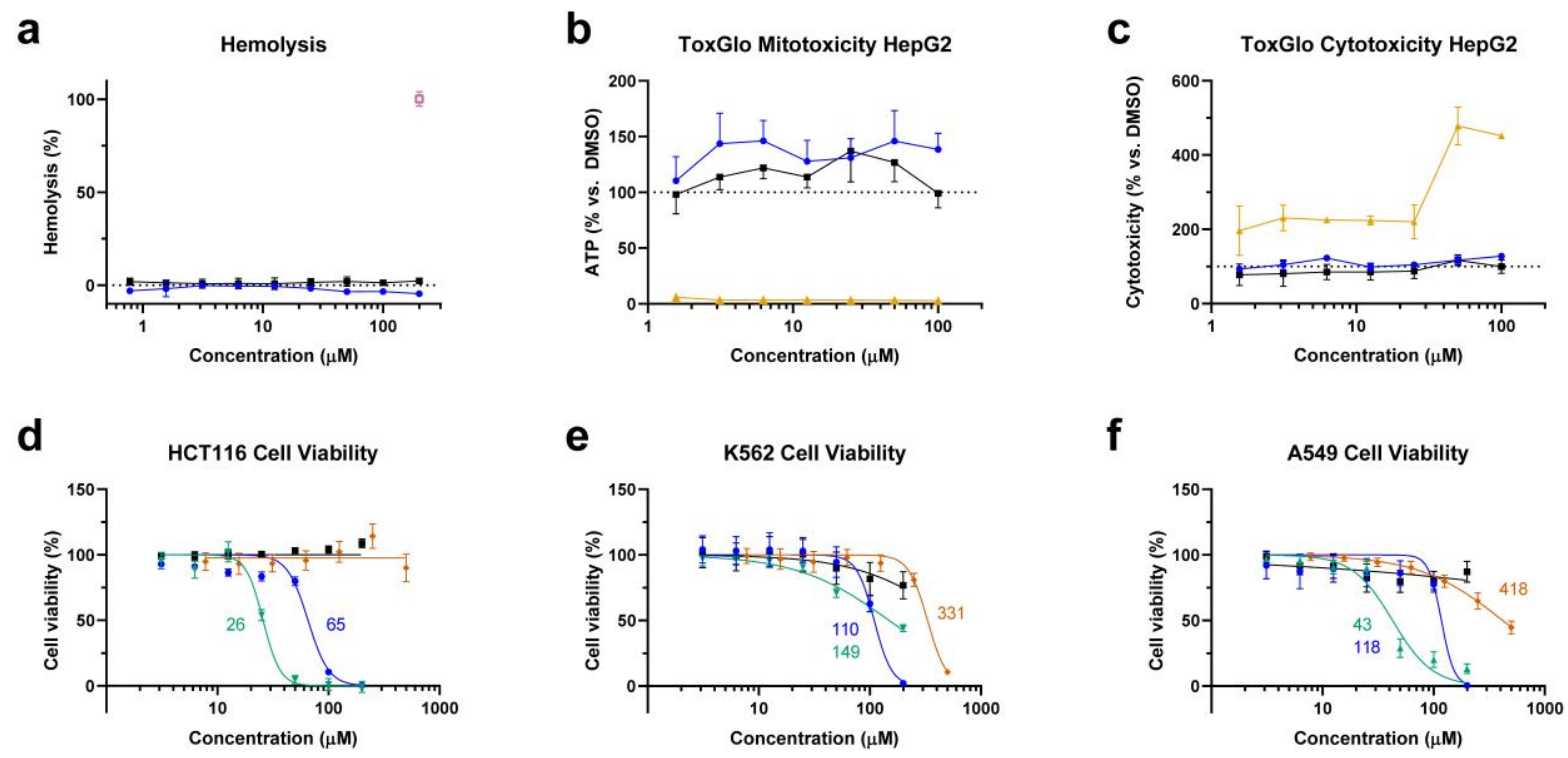

$$
\begin{array}{ll}
\rightarrow \text { OPP-3 } & \square \text { Triton X-100 } \rightarrow \text { Doxycycline } \\
\rightarrow \text { Clindamycin } & \rightarrow \text { Antimycin } \rightarrow \text { Azithromycin }
\end{array}
$$

Extended Data Figure 4 | Effects of iboxamycin (OPP-3) on mammalian cells. a, Normalized hemolysis of human erythrocytes relative to Triton X-100. b, c, Mitochondrial ToxGlo data showing effects of iboxamycin on HepG2 cellular membrane integrity and ATP production relative to vehicle-treated control (antimycin serves as a positive control for mitotoxicity). d-f, Comparison of effects of iboxamycin, clindamycin, doxycycline, and azithromycin on cell viability (CellTiter-Glo). Where applicable, GI50 values $(\mu \mathrm{M})$ are reported beside the doseresponse curves. 
Extended Data Table 1 | Pharmacokinetics of clindamycin and iboxamycin.

\begin{tabular}{|c|c|c|c|c|c|}
\hline & \multirow[b]{2}{*}{ Species } & \multicolumn{2}{|c|}{ Clindamycin } & \multicolumn{2}{|c|}{ Iboxamycin (OPP-3) } \\
\hline & & i.v. & p.o. & i.v. & p.o. \\
\hline Dose $(\mathrm{mg} / \mathrm{kg})$ & $m$ & 60 & 60 & 60 & 60 \\
\hline $\mathrm{AUC}(\mu \mathrm{g} \cdot \mathrm{h} / \mathrm{mL})$ & & $2.1 \pm 0.5$ & $0.51 \pm 0.09$ & $6.8 \pm 1.3$ & $1.6 \pm 0.4$ \\
\hline $\mathrm{C}_{\max }(\mu \mathrm{g} / \mathrm{mL})$ & & $2.9 \pm 0.7$ & $0.52 \pm 0.10$ & $7.9 \pm 3.0$ & $1.6 \pm 0.7$ \\
\hline MRT (h) & & $0.9 \pm 0.3$ & - & $1.2 \pm 0.2$ & - \\
\hline $\mathrm{CL}(\mathrm{L} / \mathrm{kg} / \mathrm{h})$ & & $29 \pm 7$ & - & $8.9 \pm 1.8$ & - \\
\hline $\mathrm{V}_{\mathrm{ss}}(\mathrm{L} / \mathrm{kg})$ & & $25 \pm 1$ & - & $12.5 \pm 0.4$ & - \\
\hline Bioavailability & & - & $24 \% \pm 7 \%$ & - & $24 \% \pm 7 \%$ \\
\hline \multirow{2}{*}{$\left.\mathrm{Clint,mic}_{\text {( }} \mu \mathrm{g} / \mathrm{min} / \mathrm{mg}\right)$} & $\mathrm{m}$ & \multicolumn{2}{|c|}{ ND } & \multicolumn{2}{|c|}{21} \\
\hline & $\mathrm{h}$ & \multicolumn{2}{|c|}{55} & \multicolumn{2}{|c|}{34} \\
\hline \multirow{2}{*}{ Plasma protein binding } & $\mathrm{m}$ & \multicolumn{2}{|c|}{$72 \%$} & \multicolumn{2}{|c|}{$95 \%$} \\
\hline & $\mathrm{h}$ & \multicolumn{2}{|c|}{$87 \%$} & \multicolumn{2}{|c|}{$92 \%$} \\
\hline
\end{tabular}

Parameters were calculated by a non-compartmental model ( $\mathrm{n}=3$ animals per study arm), and are reported as their mean \pm standard deviation, where applicable. i.v., intravenous; p.o., per os; AUC, area under the curve; MRT, mean retention time; $\mathrm{CL}$, clearance; $\mathrm{V}_{\mathrm{ss}}$, volume of distribution at steady state; $\mathrm{Cl}_{\mathrm{int} \text {,mic }}$, liver microsome intrinsic clearance; h, human; $\mathrm{m}$, mouse. 
Extended Data Table 2 | Mouse survival in S. pyogenes systemic infection model

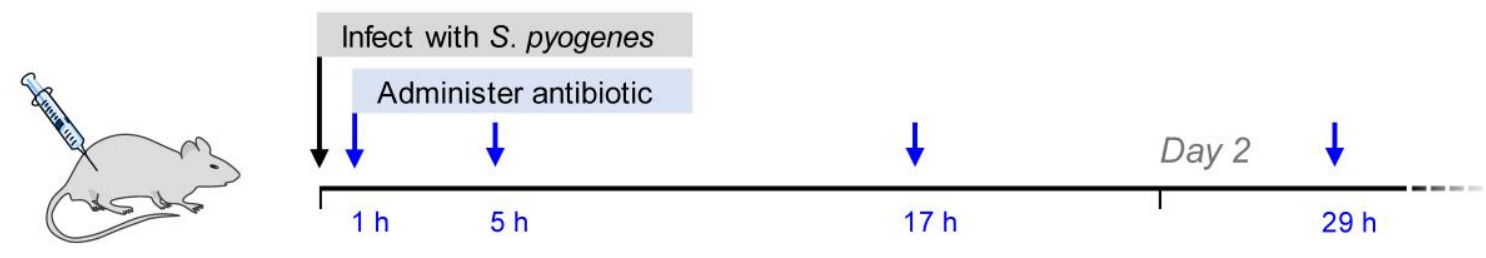

\begin{tabular}{|c|c|c|c|c|c|c|c|c|c|}
\hline & $\begin{array}{l}\text { Mice } \\
\text { surviving }\end{array}$ & Day 1 & Day 2 & Day 3 & Day 4 & Day 5 & Day 6 & Day 7 & $\mathrm{n}$ \\
\hline Vehicle $(10 \%$ & Captisol) & 10 & 3 & 2 & 2 & 2 & 1 & 1 & 10 \\
\hline \multirow[t]{3}{*}{ Clindamycin } & 1 mg/kg & 8 & 8 & 8 & 8 & 8 & 8 & 8 & 8 \\
\hline & $3 \mathrm{mg} / \mathrm{kg}$ & 8 & 8 & 8 & 8 & 8 & 8 & 8 & 8 \\
\hline & $10 \mathrm{mg} / \mathrm{kg}$ & 8 & 7 & 7 & 7 & 7 & 7 & 7 & 8 \\
\hline \multirow{3}{*}{$\begin{array}{r}\text { Iboxamycin } \\
\text { (OPP-3) }\end{array}$} & $1 \mathrm{mg} / \mathrm{kg}$ & 8 & 7 & 7 & 7 & 7 & 7 & 7 & 8 \\
\hline & $3 \mathrm{mg} / \mathrm{kg}$ & 8 & 8 & 8 & 8 & 8 & 8 & 8 & 8 \\
\hline & $10 \mathrm{mg} / \mathrm{kg}$ & 8 & 8 & 8 & 8 & 8 & 8 & 8 & 8 \\
\hline
\end{tabular}


Extended Data Table 3 | Mutations selected in $E$. coli strain SQ110DTC conferring resistance to iboxamycin (OPP-3)

\begin{tabular}{ccccc}
\hline Clone & $\begin{array}{c}\text { OPP-3 concentration } \\
\text { on selection plate }(\mu \mathrm{g} / \mathrm{mL})\end{array}$ & Mutation & OPP-3 & Clindamycin \\
\hline SQ110DTC & & WT & 0.03 & 16 \\
OPP-3R1 & 1 & A2058G & 4 & $>128$ \\
OPP-3R13 & 1 & A2059G & 2 & $>128$ \\
OPP-3R14 & 1 & A2058G & ND & ND \\
OPP-3R2 & 0.25 & A2059G & 2 & $>128$ \\
OPP-3R3 & 0.25 & A2059G & ND & ND \\
OPP-3R4 & 0.25 & A2058G & ND & ND \\
OPP-3R5 & 0.25 & A2059G & ND & ND \\
OPP-3R6 & 0.25 & A2059G & ND & ND \\
OPP-3R7 & 0.25 & A2059G & ND & ND \\
OPP-3R8 & 0.25 & A2059G & ND & ND \\
OPP-3R9 & 0.25 & A2058G & 4 & $>128$ \\
OPP-3R10 & 0.25 & A2058G & ND & ND \\
OPP-3R11 & 0.25 & A2058G & ND & ND \\
OPP-3R12 & 0.25 & A2059G & ND & ND \\
\hline
\end{tabular}



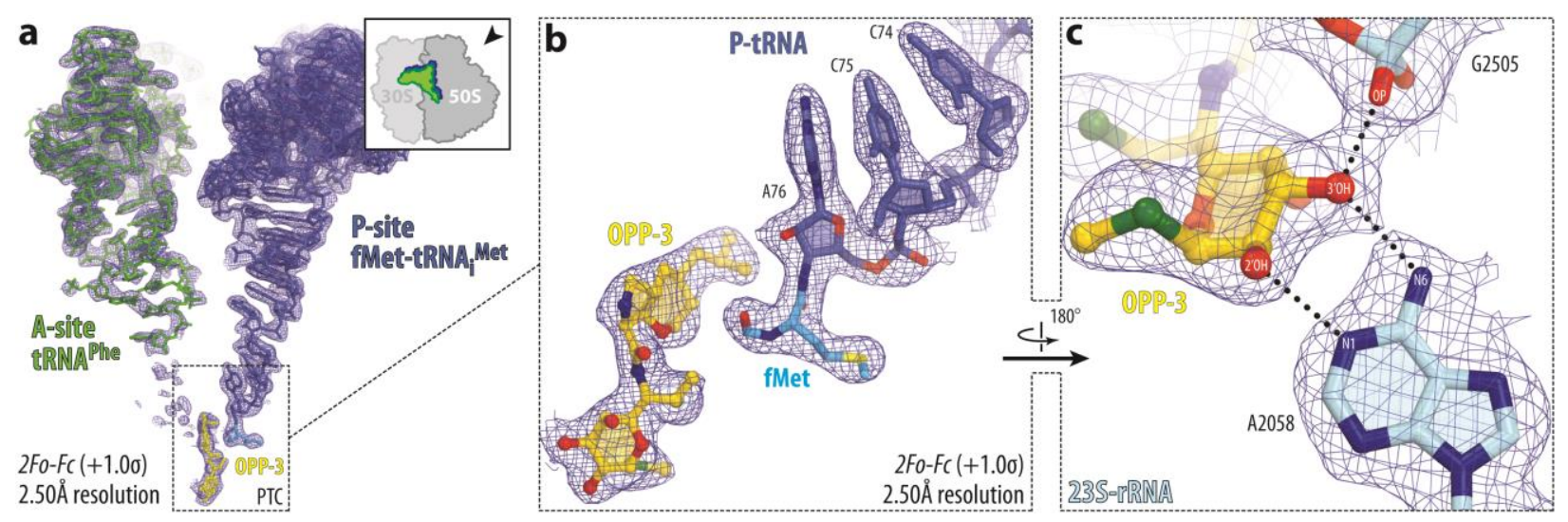

Extended Data Figure $5 \mid$ A- and P-site tRNAs in the structure of ribosome-bound iboxamycin (OPP-3). High-resolution (2.50 $\AA$ ) $2 F_{o}-F_{c}$ electron density map (blue mesh) corresponding to ribosome-bound iboxamycin (yellow), deacylated A-site tRNA ${ }^{\text {Phe }}$ (green) and aminoacylated initiator P-site fMet-NH-tRNA ${ }_{i}^{\text {Met }}$ (dark blue). The refined models of tRNAs are displayed in their respective electron-density map contoured at $1.0 \sigma$. In $\mathbf{a}$, The entire bodies of the A- and P-site tRNAs are viewed from the back of the $50 \mathrm{~S}$ subunit, as indicated by the inset. Ribosome subunits are omitted for clarity. Note that iboxamycin binding to the ribosome prevents accommodation of the aminoacyl-bearing CCA-end of the A-site tRNA. b, Close-up view of the P-site tRNA CCA-end bearing a formyl-methionyl (cyan) residue. Nitrogen atoms are colored blue; oxygen red, and sulfur yellow. c, Detailed arrangement of the hydrogen bonds formed between the lincosamine sugar of iboxamycin with the residues A2058 and G2505 of the 23S rRNA (blue). 


\section{METHODS}

\section{In vitro susceptibility}

Minimum inhibitory concentrations (MICs) were determined by the broth microdilution method or the agar dilution method following the Clinical and Laboratory Standards Institute (CLSI) guidelines ${ }^{53,54,55}$. Standard bacterial strains were obtained from American Type Culture Collection (ATCC). Other bacterial strains were obtained from hospitals and commercial sources, so noted in Supplementary Table 1. Before the start of the MIC experiment, standard and test compound stock solutions were prepared in dimethyl sulfoxide (DMSO, Aldrich D2650) at a stock concentration of 5,120 $\mu \mathrm{g} / \mathrm{mL}$. The compound concentration range typically employed for each experiment was $128-0.06 \mu \mathrm{g} / \mathrm{mL}$ (2.5\% final DMSO concentration). Clindamycin was used as a comparator in all experiments and each experiment was performed in triplicate. Specialized procedures were employed for streptococcal and anaerobic strains (see Supplementary Methods for details). All other bacterial strains were sub-cultured on blood agar plates (tryptic soy agar with $5 \%$ sheep blood, Hardy Diagnostics) and incubated overnight at $35^{\circ} \mathrm{C}$. Organisms were suspended in cation-adjusted Mueller-Hinton broth (CaMHB, BD 212322) and optical density was adjusted to $0.5 \mathrm{McFarland}$ standard. Suspensions were further diluted to obtain a final inoculum of $5 \times 10^{5} \mathrm{CFU} / \mathrm{mL}$ for broth microdilution experiments. The minimum concentration of compound required to inhibit visible bacterial growth after $24 \mathrm{~h}$ of incubation was recorded as the MIC.

\section{Time-kill studies}

Time-kill studies were performed using four different concentrations of standard and test compounds (1, 2, 4, and $10 \times \mathrm{MIC})$. Experiments were performed in duplicate following CLSI guidelines. An inoculum was prepared in Mueller-Hinton broth (MHB) containing 0.5$5 \times 10^{6} \mathrm{CFU} / \mathrm{mL}$ of test organism. Cultures were incubated at $37{ }^{\circ} \mathrm{C}$ in a shaker incubator at 110 
rpm; a flask containing bacteria left unexposed to antibiotic was used as untreated control. At time points $0,1,3,6$, and $24 \mathrm{~h}$ following administration of antibiotic, bacterial counts were determined from each flask by serial dilution and plating on brain heart infusion agar (BD, Sparks, MD). Plates were incubated at $37{ }^{\circ} \mathrm{C}$ in incubators for $18-24$ hours to determine bacterial counts. For streptococcal strains, blood agar plates (Tryptic Soy Agar with 5\% Sheep Blood) were used for culturing the organisms (plates were incubated in the presence of $5 \% \mathrm{CO}_{2}$ ), and time-kill studies were performed in media supplemented with 5\% laked horse blood. Compounds exhibiting $\geq 3 \log _{10} \mathrm{CFU} / \mathrm{mL}$ reduction compared to initial counts are considered bactericidal. Compounds exhibiting $\leq 2 \log _{10} \mathrm{CFU}$ reduction, or which maintain counts similar to the initial bacterial counts, are considered bacteriostatic.

\section{Post-antibiotic and post-antibiotic sub-MIC effect}

Measurements of PAE and PA-SME durations were performed according to the methods described by Odenholt-Tornqvist and co-workers ${ }^{56}$. Bacterial strains were cultured overnight on blood agar plates. Optical density was adjusted to $0.5 \mathrm{McFarland}$ standard in CaMHB and suspensions were diluted further into 50-mL sterile Erlenmeyer flasks containing $20 \mathrm{~mL}$ CaMHB to achieve a bacterial load of $0.5-1 \times 10^{6} \mathrm{CFU} / \mathrm{mL}$. Cultures were then exposed to test and standard antibiotic at 1,2, 4, and $10 \times$ MIC concentrations for 1 hour at $37^{\circ} \mathrm{C}$ in a shaker incubator at $110 \mathrm{rpm}$ (0.5\% final DMSO concentration). A flask containing the unexposed bacterial strain was left as untreated control. Post-exposure, $100 \mu \mathrm{L}$ of bacterial culture was diluted in $900 \mu \mathrm{L}$ of sterile CaMHB; $100 \mu \mathrm{L}$ of this diluted sample was immediately diluted further in $19.9 \mathrm{~mL}$ of sterile CaMHB (1:2,000 final dilution). For PA-SME studies, post exposure and dilution, each flask (except those in the control group) was supplemented with 0.25 and $0.5 \times$ MIC per $\mathrm{mL}$ of specific antibiotic ( $0.5 \%$ final DMSO concentration). Flasks were incubated at $37{ }^{\circ} \mathrm{C}$ in a shaker incubator 
(110 rpm). Samples (100 $\mu \mathrm{L}$ from each flask per time point) were collected at $0,1,3,6$, and $24 \mathrm{~h}$ from each flask and were serially diluted. From each dilution, $100 \mu \mathrm{L}$ and $10 \mu \mathrm{L}$ from each dilution were plated in duplicate on TSA plates. Plates were incubated overnight at $35^{\circ} \mathrm{C}$ to determine the bacterial counts (limit of detection $=20 \mathrm{CFU} /$ plate or $2 \times 10^{2} \mathrm{CFU} / \mathrm{mL}$ ). These counts were plotted against time with linear interpolation between time points to obtain the growth-kinetic curves. PAE and PA-SME were determined from these curves using the formulae:

$$
\mathrm{PAE}=T-C
$$

where $T$ is the time required for bacteria previously exposed to antibiotic to multiply $1 \log _{10}$ above counts immediately following dilution, and $C$ is the corresponding time required for untreated culture to do the same; and

$$
\mathrm{PA}-\mathrm{SME}=T_{p a}-C
$$

where $T_{p a}$ is the time taken for bacteria previously exposed to antibiotic and then re-exposed to sub-MIC concentrations to increase $1 \log _{10}$ above counts immediately following dilution.

\section{Mammalian cell experiments}

All compounds were dissolved in sterile DMSO to a stock concentration of $20 \mu \mathrm{M}$ and aliquoted prior to freezing at $-20^{\circ} \mathrm{C}$. Aliquots were limited to a maximum of 3 freeze-thaw cycles. A549 (human pulmonary carcinoma; male) cells were cultured in Dulbecco's Modified Eagle Medium (DMEM) supplemented with 10\% fetal bovine serum. K562 (human chronic myelogenous leukemia; female) cells were cultured in Iscove's Modification of DMEM (IMDM) media supplemented with 10\% fetal bovine serum. HCT116 (human colorectal carcinoma; male) cells were cultured in McCoy's 5a (Iwakata \& Grace Modification) media supplemented with 10\% fetal bovine serum. HepG2 (human hepatocellular carcinoma; male) cells were cultured in Eagle's 
Minimum Essential Medium (EMEM) supplemented with 10\% fetal bovine serum. All cell cultures were maintained in a $5 \% \mathrm{CO}_{2}$ incubator at $37^{\circ} \mathrm{C}$.

Growth inhibition was studied using the Promega CellTiter-Blue cell viability assay pursuant to the manufacturer's protocol. Briefly, cells were harvested, diluted, and mixed with the desired concentration of test compound. This suspension was then added to a 96-well plate ( $5 \times 10^{4}$ cells/mL, $100 \mu \mathrm{L} /$ well, 5 replicates per test compound), and the plates were incubated for $72 \mathrm{~h}$ at $37{ }^{\circ} \mathrm{C}$ in a $5 \% \mathrm{CO}_{2}$ incubator. Next, $20 \mu \mathrm{L}$ of CellTiter-Blue reagent was added to each well and the plates were incubated for $4 \mathrm{~h}$. Plates were read on a SpectraMax i3 plate reader. $\mathrm{GI}_{50}$ values were determined by nonlinear regression using GraphPad Prism and represent the mean of at least three independent experiments.

Mitochondrial toxicity assays were conducted using the Promega Mitochondrial ToxGlo assay, pursuant to the manufacturer's protocol.

\section{Animal studies}

Animal experiments were performed at the Biology Research Infrastructure Laboratory of Harvard University following Institutional Animal Care and Use Committee (IACUC)-approved protocols. Animals were maintained in accordance with the recommendations in the Guide for the Care and Use of Laboratory Animals of the National Institutes of Health. Pathogen-free, 5-6week-old, female CD-1 mice weighing 22-26 grams were obtained from Charles River Laboratories, Inc. (Kingston, NY). Animals were acclimated for a minimum of three days prior to start of the studies. Mice were caged as a group of four per cage, were housed at $21-23{ }^{\circ} \mathrm{C}$ with humidity ranging from 30-70\%, were exposed to 12-hour light and dark cycles, and were supplied with food and water ad libitum.

\section{Mouse thigh infection studies}


Mice were rendered neutropenic by administering cyclophosphamide intraperitoneally, $150 \mathrm{mg} / \mathrm{kg}$ four days prior, and $100 \mathrm{mg} / \mathrm{kg}$ one day prior to infection. Bacterial strains were cultured overnight on blood agar plates at $35^{\circ} \mathrm{C}$ in $5 \% \mathrm{CO}_{2}$. Bacterial inoculum was prepared in sterile brain heart infusion broth (BHIB) and OD was adjusted to 0.1 at $600 \mathrm{~nm}$; the inoculum was further diluted to achieve a bacterial load of $0.5-1 \times 10^{7} \mathrm{CFU} / \mathrm{mL}$. Animals were infected by intramuscular injection of $0.1 \mathrm{~mL}$ of inoculum into each thigh $(0.2 \mathrm{~mL}$ of inoculum was used per animal). Mice were divided into 4 groups: iboxamycin ( $\mathrm{n}=12$ animals), clindamycin $(\mathrm{n}=12)$, vehicle-treated control $(n=12)$, and untreated baseline $(n=2)$. At $2 \mathrm{~h}$ post-infection $(\mathrm{t}=0)$, the untreated animals were euthanized to obtain baseline bacterial counts; the others received a single dose of iboxamycin $(6 \mathrm{mg} / \mathrm{mL}$ in $10 \%$ Captisol, $250 \mu \mathrm{L}, 60 \mathrm{mg} / \mathrm{kg})$, clindamycin phosphate $(6 \mathrm{mg} / \mathrm{mL}$ in sterile saline, $250 \mu \mathrm{L}, 60 \mathrm{mg} / \mathrm{kg})$, or sterile vehicle (10\% Captisol, $250 \mu \mathrm{L})$ by intraperitoneal injection. Intramuscular bacterial counts in treated animals were determined 1, 3, $6,12,18$, and $24 \mathrm{~h}$ after treatment. At each time point animals from each group $(\mathrm{n}=2)$ were euthanized by $\mathrm{CO}_{2}$ inhalation, and thighs ( 4 per time point) were aseptically removed and homogenized in sterile saline. Homogenates were serially diluted and plated on blood agar plates (each sample was plated in duplicate), and bacterial counts ( $\log _{10} \mathrm{CFU} /$ thigh) were determined after incubating the plates overnight at $35{ }^{\circ} \mathrm{C}$ in $5 \% \mathrm{CO}_{2}$. Each experiment was repeated twice, and mean values were calculated ( $n=16$ counts across 4 independent mice per time point).

\section{Mouse systemic infection study}

S. pyogenes ATCC 19615 was cultured overnight on blood agar plates in $5 \% \mathrm{CO}_{2}$ at $35^{\circ} \mathrm{C}$ before being suspended in BHIB. The optical density of the bacterial suspension was adjusted to 0.1 at $600 \mathrm{~nm}$ using sterile BHIB and was then further diluted 1:1 in 10\% hog gastric mucin (HGM, type III) to prepare the infecting inoculum in 5\% HGM. Mice were infected by intraperitoneal 
injection of $250 \mu \mathrm{L}$ infecting inoculum, representing $1-2 \times 10^{6} \mathrm{CFU}$ per animal. At time points 1 , 5,17 , and 29 h post-infection, animals were administered intravenous infusions of iboxamycin (1, 3 , or $10 \mathrm{mg} / \mathrm{kg}, 250 \mu \mathrm{L}$ in $10 \%$ Captisol; 8 mice per dose level), clindamycin phosphate $(1,3$, or $10 \mathrm{mg} / \mathrm{kg}, 250 \mu \mathrm{L}$ in normal saline; 8 mice per dose level), or vehicle as negative control (10\% Captisol, $250 \mu \mathrm{L} ; 10$ mice). Animals were monitored for survival for seven days following infection.

\section{Search for spontaneous resistance in standard bacterial strains}

Cation-adjusted Mueller-Hinton agar plates containing iboxamycin or clindamycin at concentrations corresponding to $4 \times \mathrm{MIC}, 8 \times \mathrm{MIC}$, and $16 \times \mathrm{MIC}$ were prepared. Bacterial strains (S. aureus ATCC 29213, S. aureus MRSA, and E. faecalis ATCC 29212) were suspended in normal saline and the optical density was adjusted to 1.50 at $600 \mathrm{~nm}$. Bacterial counts were measured by serial dilution and plating. Bacterial inoculum $(250 \mu \mathrm{L})$ was transferred to the media plate and was spread evenly. Plates were incubated at $37^{\circ} \mathrm{C}$ for 3 days and were observed daily for resistant colonies to emerge. No colonies were noted on any plate during the course of this experiment.

\section{Selection of iboxamycin resistant mutants in SQ110DTC}

The MICs of iboxamycin and clindamycin against $E$. coli strain SQ110DTC ${ }^{45}$ were determined in lysogeny broth (LB) medium by placing $100 \mu \mathrm{L}$ of exponentially growing cells in the wells of a 96-well plate, adding 2-fold dilutions of antibiotics and incubating plates for $18 \mathrm{~h}$ at $37^{\circ} \mathrm{C}$ (Extended Data Table 3). For selection of the resistant mutants, $\sim 10^{9}$ colony forming units (1.2 mL of the exponentially growing cell culture with optical density of $\left.A_{600}=1.2\right)$, were plated on LB/agar plates containing either $0.25 \mu \mathrm{g} / \mathrm{mL}$ or $1 \mu \mathrm{g} / \mathrm{mL}$ of iboxamycin $(\sim 10 \times$ or $\sim 30 \times \mathrm{MIC}$, respectively). Approximately 20 colonies appeared on both plates after incubation at $37{ }^{\circ} \mathrm{C}$ for 
$24 \mathrm{~h}$. The segment of the $23 \mathrm{~S}$ rRNA gene corresponding to domains V and VI of the 23S rRNA was PCR-amplified from 14 randomly selected colonies using the primers 2020R (CCC GAG ACT CAG TGA AAT TGA ACT C) and L2904 (AAG GTT AAG CCT CAC GG). PCR products were sequenced using the primer L2667 (GGT CCT CTC GTA CTA GGA GCA G).

\section{Toeprinting analysis}

The ermBL DNA template for toeprinting was generated by a 4-primer cross-over PCR using primers T7, NV1, ermB3-F and ermB3-R. The ermDL template was generated in the same way using primers RLR-fwd, RLR-rev, T7-SD-fwd and T7-SD-rev. In vitro translation in the PURExpress system (New England Biolabs) and toeprinting analysis were carried out as described

previously ${ }^{57}$. The antibiotics (mupirocin, clindamycin, erythromycin, iboxamycin) were present in the reactions at a final concentration of $50 \mu \mathrm{g} / \mathrm{mL}$.

Primer sequences used for toeprinting are as follows: T7 (TAA TAC GAC TCA CTA TAG GG); NV1 (GGT TAT AAT GAA TTT TGC TTA TTA AC); ermB3-F (TAA TAC GAC TCA CTA TAG GGC TTA AGT ATA AGG AGG AAA AAA TAT GTT GGT ATT CCA AAT GCG TAA TGT AGA TAA AAC ATC TAC); ermB3-R (GGT TAT AAT GAA TTT TGC TTA TTA ACG ATA GAA TTC TAT CAC TTA TTT CAA AAT AGT AGA TGT TTT ATC TAC ATT ACG); RLR-fwd (GGA GGA AAA AAT ATG ACA CAC TCA ATG AGA CTT CGT ATT TTC CC); RLR-rev (CTA TCA CTT ACA AAG TTG GGA AAA TAC GAA GTC TCA TTG AG); T7-SD-fwd (TAA TAC GAC TCA CTA TAG GGC TTA AGT ATA AGG AGG AAA AAA TAT GAC ACA CTC AAT G); T7-SD-rev (GGT TAT AAT GAA TTT TGC TTA TTA ACG ATA GAA TTC TAT CAC TTA CAA AGT TGG GAA AAT).

Crystallization of iboxamycin in complex with WT and Erm-modified Tth 70S ribosomes 
Wild-type 70S ribosomes from Thermus thermophilus (strain HB8) containing unmodified residue A2058 of the 23S rRNA were prepared as described previously ${ }^{50}$. Purification of the Ermmodified 70S ribosomes from T. thermophilus (strain HB27 expressing Erm-like enzyme from Bifidobacterium thermophilum) containing N6-dimethylated A2058 residue in the 23S rRNA was accomplished as optimized previously for the wild-type $70 \mathrm{~S}$ ribosomes from $T$. thermophilus ${ }^{52}$. Deacylated tRNA ${ }^{\text {Phe }}$ and non-hydrolyzable aminoacylated fMet-NH-tRNA ${ }_{i}^{\text {Met }}$ were prepared as described previously ${ }^{49,58}$. Complexes of the A2058-dimethylated or unmethylated Tth 70S ribosomes with mRNA, deacylated A-site tRNA ${ }^{\text {Phe }}$, and P-site hydrolysis-resistant aminoacylated fMet-NH-tRNA ${ }_{i}^{\text {Met }}$ were formed as described previously ${ }^{49,50}$. For Tth 70S ribosome complexes with iboxamycin, the antibiotic was included in the crystallization mixture (250 $\mu \mathrm{M}$ each) and then later added to the stabilization buffers $(250 \mu \mathrm{M}$ each). Collection and processing of the X-ray diffraction data, model building, and structure refinement were performed as described in our previous publications $50,52,59,60,61$. Structural models and restraints for iboxamycin were generated using PRODRG online software (http://prodrg1.dyndns.org) ${ }^{62}$. The statistics of data collection and refinement are compiled in Supplementary Table 2. All figures showing atomic models were generated using PyMol software (www.pymol.org).

\section{Statistical analyses}

Statistical analysis was performed using GraphPad Prism. Mouse thigh-infection study data (24-h time points) were compared using a one-way ANOVA followed by Tukey's multiple comparisons test. 
SUPPLEMENTARY METHODS

Chemical synthesis of oxepanoprolinamide analogs

SUPPLEMENTARY TABLES

Supplementary Table 1 | In vitro antibacterial activity of iboxamycin

Supplementary Table 2 | X-ray crystallographic data for iboxamycin-ribosome complexes

\section{SUPPLEMENTARY VIDEO}

Supplementary Video | Illustration of iboxamycin binding to the wild-type ribosome 


\section{REFERENCES}

${ }^{1}$ Scientific Roadmap for Antibiotic Discovery. Pew Charitable Trusts (11 May 2016); http://www.pewtrusts.org/antibiotic-discovery

${ }^{2}$ Wright, P.M., Seiple, I.B. \& Myers, A.G. The Evolving Role of Chemical Synthesis in Antibacterial Drug Discovery. Angew. Chem. Int. Ed. 53, 8840-8869 (2014).

3 Charest, M.G., Lerner, C.D., Brubaker, J.D., Siegel, D.R. \& Myers, A.G. A Convergent Enantioselective Route to Structurally Diverse 6-Deoxytetracycline Antibiotics. Science 308, 395-398 (2005).

4 Seiple, I.B. et al. A platform for the discovery of new macrolide antibiotics. Nature 533, 338345 (2016).

$5 \mathrm{Li}, \mathrm{Q}$. et al. Synthetic group A streptogramin antibiotics that overcome Vat resistance. Nature 586, 145-150 (2020).

${ }^{6}$ Myers, A \& Clark, R.B. Discovery of Macrolide Antibiotics Effective Against Multi-Drug Resistant Gram-Negative Pathogens. Acc. Chem. Res. (2021). In press.

${ }^{7}$ Wilson, D.N. Ribosome-targeting antibiotics and mechanisms of bacterial resistance. Nat. Rev. Microbiol. 12, 35-48 (2014).

${ }^{8}$ Lin, J., Zhou, D., Steitz, T.A., Polikanov, Y.S. \& Gagnon, M.G. Ribosome-Targeting Antibiotics: Modes of Action, Mechanisms of Resistance, and Implications for Drug Design. Annu. Rev. Biochem. 87, 451-478 (2018).

${ }^{9}$ Mason, D.J., Dietz, A. \& De Boer, C. Lincomycin, a new antibiotic. I. Discovery and biological properties. Antimicrob. Agents Chemother. 554-559 (1962)

${ }^{10}$ Birkenmeyer, R.D. \& Kagan, F. Lincomycin. XI. Synthesis and structure of clindamycin. A potent antibacterial agent. J. Med. Chem. 13, 616-619 (1970).

${ }^{11}$ Phillips, I. Past and current use of clindamycin and lincomycin. J. Antimicrob. Chemother. 7 (Suppl A) 11-18, (1981).

12 Birkenmeyer, R.D., Kroll, S.J., Lewis, C., Stern, K.F. \& Zurenko, G.E. Synthesis and antimicrobial activity of clindamycin analogs: pirlimycin, a potent antibacterial agent. $J$. Med. Chem. 27, 216-223 (1984). 
${ }^{13}$ O’Dowd, H., Erwin, A.L. \& Lewis, J.G. Lincosamide Antibacterials. In Natural Products in Medicinal Chemistry; Hanessian, S., Ed.; Wiley-VCH Verlag GmbH \& Co. KGaA, Weinheim, Germany (2014).

${ }^{14}$ Lewis, J.G., Anandan, S.K., O’Dowd, H., Gordeev, M.F. \& Li, L. Lincomycin derivatives possessing antibacterial activity. US Patent 7,361,743 B2 (2005)

${ }^{15}$ Wakiyama, Y. et al. Synthesis and SARs of novel lincomycin derivatives Part 5: optimization of lincomycin analogs exhibiting potent antibacterial activities by chemical modification at the 6- and 7-positions. J. Antibiot. (Tokyo) 71, 298-317 (2018).

${ }^{16}$ Hirai, Y. et al. Characterization of compound A, a novel lincosamide derivative active against methicillin-resistant Staphylococcus aureus. J. Antibiot. 74, 124-132 (2021).

${ }^{17}$ Umemura, E. et al. Lincosamide derivatives and antimicrobial agents comprising the same as active ingredient. US Patent 7,867,980 B2 (2008).

${ }^{18}$ Sutcliffe, J. \& Leclercq, R. Mechanisms of resistance to macrolides, lincosamides, and ketolides. in Macrolide Antibiotics; Schönfeld, W. \& Kirst, H.A., Eds.; Birkhäuser Verlag AG, Basel, Switzerland (281-318, 2002).

${ }^{19}$ Roberts, M.C. et al. Nomenclature for Macrolide and Macrolide-Lincosamide-Streptogramin B Resistance Determinants. Antimicrob. Agents Chemother. 43, 2823-2830 (1999).

${ }^{20}$ Skinner, R., Cundliffe, E. \& Schmidt, F.J. Site of action of a ribosomal RNA methylase responsible for resistance to erythromycin and other antibiotics. J. Biol. Chem. 258, 1270212706 (1983).

${ }^{21}$ Lai, C.J. \& Weisblum, B. Altered Methylation of Ribosomal RNA in an Erythromycin-Resistant Strain of Staphylococcus aureus. Proc. Natl. Acad. Sci. USA 68, 856-860 (1971).

${ }^{22}$ Griffith, L.J., Ostrander, W.E., Mullins, C.G. \& Beswick, D.E. Drug antagonism between lincomycin and erythromycin. Science 147, 746-747 (1965).

${ }^{23}$ CDC. Antibiotic Resistance Threats in the United States, 2019. Atlanta, GA: U.S. Department of Health and Human Services, CDC. Available online at www.cdc.gov/DrugResistance/Biggest-Threats.html (2019). 
${ }^{24}$ Toh, S.M. et al. Acquisition of a natural resistance gene renders a clinical strain of methicillinresistant Staphylococcus aureus resistant to the synthetic antibiotic linezolid. Mol. Microbiol. 64, 1506-1514 (2007).

25 Long, K.S., Poehlsgaard, J., Kehrenberg, C., Schwarz, S. \& Vester, B. The Cfr rRNA Methyltransferase Confers Resistance to Phenicols, Lincosamides, Oxazolidinones, Pleuromutilins, and Streptogramin A Antibiotics. Antimicrob. Agents Chemother. 50, 2500-2505 (2006).

${ }^{26}$ Giessing, A.M. et al. Identification of 8-methyladenosine as the modification catalyzed by the radical SAM methyltransferase Cfr that confers antibiotic resistance in bacteria. RNA $\mathbf{1 5}$, 327-336 (2009).

${ }^{27}$ Smith, L.K. \& Mankin, A.S. Transcriptional and Translational Control of the mlr Operon, Which Confers Resistance to Seven Classes of Protein Synthesis Inhibitors. Antimicrob. Agents Chemother. 52, 1703-1712 (2008).

${ }^{28}$ Mitcheltree, M.J., Stevenson, J.W., Pisipati, A. \& Myers, A.G. A Practical, Component-Based Synthetic Route to Methylthiolincosamine Permitting Facile Northern-Half Diversification of Lincosamide Antibiotics. In review (2021).

29 Mitcheltree, M.J. A Platform for the Discovery of New Lincosamide Antibiotics. PhD Dissertation, Harvard University (2018).

${ }^{30}$ Silvestre, K.J. Design, Synthesis, and Study of Lincosamide Antibiotics Containing a Bicyclic Amino Acid Moiety. PhD Dissertation, Harvard University (2019).

${ }^{31}$ Moga, I. Novel Lincosamide Antibiotics Containing an Azepane Amino Acid Moiety. PhD Dissertation, Harvard University (2019).

${ }^{32}$ Dunkle, J.A., Xiong, L., Mankin, A.S. \& Cate, J.H. Structures of the Escherichia coli ribosome with antibiotics bound near the peptidyl transferase center explain spectra of drug action. Proc. Natl. Acad. Sci. USA 107, 17152-17157 (2010).

${ }^{33}$ Seiple, I.B., Mercer, J.A., Sussman, R.J., Zhang, Z. \& Myers, A.G. Stereocontrolled Synthesis of syn- $\beta$-Hydroxy- $\alpha$-Amino Acids by Direct Aldolization of Pseudoephenamine Glycinamide. Angew. Chem. Int. Ed. Engl. 53, 4642-4647 (2014).

${ }^{34}$ Kingsbury, J.S., Harrity, J.P.A., Bonitatebus, P.J., Jr. \& Hoveyda, A.H. A Recyclable Ru-Based Metathesis Catalyst. J. Am. Chem. Soc. 121, 791-799 (1999). 
${ }^{35}$ Magerlein, B.J. \& Kagan, F. Lincomycin. 8. 4'-Alkyl-1'-demethyl-4'-depropylclindamycins, potent antibacterial and antimalarial agents. J. Med. Chem. 12, 780-784 (1969).

${ }^{36}$ Morandi, B. Wickens, Z.K. \& Grubbs, R.H. Regioselective Wacker Oxidation of Internal Alkenes: Rapid Access to Functionalized Ketones Facilitated by Cross-Metathesis. Angew. Chem. Int. Ed. 52, 9751-9754 (2013).

${ }^{37}$ Scheiper, B., Bonnekessel, M., Krause, H. \& Fürstner, A. Selective Iron-Catalyzed CrossCoupling Reactions of Grignard Reagents with Enol Triflates, Acid Chlorides, and Dichloroarenes. J. Org. Chem. 69, 3943-3949 (2004).

${ }^{38}$ Mason, J.D., Terwilliger, D.T., Pote, A.R. \& Myers, A.G. Practical Synthesis of Iboxamyin, a Potent Antibiotic Candidate, in Amounts Suitable for Studies in Animal Infection Models. In review (2021).

${ }^{39}$ Zhao, C. et al. Investigation of Antibiotic Resistance, Serotype Distribution, and Genetic Characteristics of 164 Invasive Streptococcus pneumoniae from North China Between April 2016 and October 2017. Infect. Drug Resist. 13, 2117-2128 (2020).

${ }^{40}$ Song, J.H. et al. High Prevalence of Antimicrobial Resistance Among Clinical Streptococcus pneumoniae Isolates in Asia (an ANSORP study). Antimicrob. Agents Chemother. 48, 2101-2107 (2004).

41 Weiner-Lastinger, L.M. et al. Antimicrobial-resistant pathogens associated with adult healthcare-associated infections: Summary of data reported to the National Healthcare Safety Network, 2015-2017. Infect. Control Hosp. Epidemiol. 41, 1-18 (2020).

42 O'Shea, R. \& Moser, H.E. Physicochemical Properties of Antibacterial Compounds: Implications for Drug Discovery. J. Med. Chem. 51, 2871-2878 (2008).

${ }^{43}$ Niles, A.L. et al. A homogeneous assay to measure live and dead cells in the same sample by detecting different protease markers. Anal. Biochem. 366, 197-206 (2007).

${ }^{44}$ Marroquin, L.D., Hynes, J., Dykens, J.A., Jamieson, J.D. \& Will, Y. Circumventing the Crabtree Effect: Replacing Media Glucose with Galactose Increases Susceptibility of HepG2 Cells to Mitochondrial Toxicants. Toxicol. Sci. 97, 539-547 (2007).

${ }^{45}$ Orelle, C. et al. Tools for Characterizing Bacterial Protein Synthesis Inhibitors. Antimicrob. Agents Chemother. 57, 5994-6004 (2013). 
${ }^{46}$ Hartz, D., McPheeters, D.S., Traut, R. \& Gold, L. Extension inhibition analysis of translation initiation complexes. in Methods in Enzymology 164; Noller, H. Jr. \& Moldave, K., Eds.; Academic Press, Cambridge, Massachusetts, United States of America (419-425, 1988).

${ }^{47}$ Orelle, C. et al. Identifying the targets of aminoacyl-tRNA synthetase inhibitors by primer extension inhibition. Nucleic Acids Res. 41, e144 (2013).

${ }^{48}$ Tu, D., Blaha, G., Moore, P.B. \& Steitz, T.A. Structures of MLS $\mathrm{B}_{\mathrm{B}} \mathrm{K}$ Antibiotics Bound to Mutated Large Ribosomal Subunits Provide a Structural Explanation for Resistance. Cell 121, 257-270 (2005).

49 Polikanov, Y.S., Steitz, T.A. \& Innis, C.A. A proton wire to couple aminoacyl-tRNA accommodation and peptide-bond formation on the ribosome. Nat. Struct. Mol. Biol. 21, 787-793 (2014).

${ }^{50}$ Polikanov, Y.S., Melnikov, S.V., Soll, D. \& Steitz, T.A. Structural insights into the role of rRNA modifications in protein synthesis and ribosome assembly. Nat. Struct. Mol. Biol. 22, 342344 (2015).

${ }^{51}$ Meydan, S. et al. Retapamulin-Assisted Ribosome Profiling Reveals the Alternative Bacterial Proteome. Mol. Cell 74, 481-493 (2019).

${ }^{52}$ Svetlov, M.S. et al. Structure of Erm-modified 70S ribosome reveals the mechanism of macrolide resistance. Nat. Chem. Biol. (2021).

${ }^{53}$ CLSI. Methods for Dilution Antimicrobial Susceptibility Tests for Bacteria That Grow Aerobically; Approved Standard - Eleventh Edition. CLSI document M07-A11. Wayne, PA: Clinical and Laboratory Standards Institute (2018).

${ }^{54}$ CLSI. Methods for Antimicrobial Susceptibility Testing of Anaerobic Bacteria; Approved Standard -Eighth Edition. CLSI document M11-A8. Wayne, PA: Clinical and Laboratory Standards Institute; 2012.

${ }^{55}$ CLSI. Performance Standards for Antimicrobial Susceptibility Testing-27th Edition: CLSI supplement M100-S27. Wayne, PA: Clinical and Laboratory Standards Institute; 2017.

56 Odenholt-Tornqvist, I. Löwdin, E., Cars, O. Pharmacodynamic Effects of Subinhibitory Concentrations of $\beta$-Lactam Antibiotics In Vitro. Antimicrob. Agents Chemother. 35, 1834-1839 (1991). 
${ }^{57}$ Orelle, C. et al. Identifying the targets of aminoacyl-tRNA synthetase inhibitors by primer extension inhibition. Nucleic Acids Res. 41 e144 (2013).

${ }^{58}$ Gamper H. \& Hou, Y.M. tRNA 3'-amino-tailing for stable amino acid attachment. RNA 24, 1878-1885 (2018).

${ }^{59}$ Khabibullina, N.F. et al. Structure of Dirithromycin Bound to the Bacterial Ribosome Suggests New Ways for Rational Improvement of Macrolides. Antimicrob. Agents Chemother. 63, e02266-18 (2019).

${ }^{60}$ Almutairi, M.M. et al. Co-produced natural ketolides methymycin and pikromycin inhibit bacterial growth by preventing synthesis of a limited number of proteins. Nucleic Acids Res. 45, 9573-9582 (2017).

${ }^{61}$ Svetlov, M.S. et al. High-resolution crystal structures of ribosome-bound chloramphenicol and erythromycin provide the ultimate basis for their competition. RNA 25, 600-606 (2019).

${ }^{62}$ Schuttelkopf, A.W. \& van Aalten, D.M.F. PRODRG: a tool for high-throughput crystallography of protein-ligand complexes. Acta Crystallogr. D Biol. Crystallogr. 60, 1355-1363 (2004). 